\title{
Parallel Lemniscal and Non-Lemniscal Sources Control Auditory Responses in the Orbitofrontal Cortex (OFC)
}

\author{
Hemant K. Srivastava, ${ }^{1}$ and Sharba Bandyopadhyay ${ }^{1,2}$
}

https://doi.org/10.1523/ENEURO.0121-20.2020

\begin{abstract}
${ }^{1}$ Advanced Technology Development Centre, Indian Institute of Technology Kharagpur, Kharagpur 721302, India and ${ }^{2}$ Department of Electronics and Electrical Communication Engineering, Indian Institute of Technology Kharagpur, Kharagpur 721302, India
\end{abstract}

\begin{abstract}
The orbitofrontal cortex (OFC) controls flexible behavior through stimulus value updating based on stimulus outcome associations, allowing seamless navigation in dynamic sensory environments with changing contingencies. Sensory cue driven responses, primarily studied through behavior, exist in the OFC. However, OFC neurons' sensory response properties, particularly auditory, are unknown in the mouse, a genetically tractable animal. We show that mouse OFC single neurons have unique auditory response properties showing pure oddball detection and long timescales of adaptation resulting in stimulus-history dependence. Further, we show that OFC auditory responses are shaped by two parallel sources in the auditory thalamus, lemniscal and non-lemniscal. The latter underlies a large component of the observed oddball detection and additionally controls persistent activity in the OFC through the amygdala. The deviant selectivity can serve as a signal for important changes in the auditory environment. Such signals, if coupled with persistent activity, obtained by disinhibitory control from the non-lemniscal auditory thalamus or amygdala, will allow for associations with a delayed outcome related signal, like reward prediction error, and potentially forms the basis of updating stimulus outcome associations in the OFC. Thus, the baseline sensory responses allow the behavioral requirementbased response modification through relevant inputs from other structures related to reward, punishment, or memory. Thus, alterations in these responses in neurologic disorders can lead to behavioral deficits.
\end{abstract}

Key words: deviant detection; lemniscal non-lemniscal auditory pathways; persistent activity; stimulus history dependence; stimulus outcome association

\section{Significance Statement}

Orbitofrontal cortex (OFC) has been shown to influence the stimulus representation in the sensory cortices allowing them to adjust to the changing contingencies in the environment, but how OFC itself receives and engages with the incoming stimulus is poorly understood. The response properties of the OFC neurons from a sensory perspective, independent of behavioral state and other cognitive processes, are not known. We show that OFC robustly responds to auditory stimulation with strong context dependence and selectivity to oddball or deviant stimuli. We also show that both lemniscal and non-lemniscal pathways, both at cortical and subcortical levels, differentially contribute to auditory responses in the OFC.

Received March 28, 2020; accepted May 10, 2020; First published August 4, 2020.

The authors declare no competing financial interests.
Author contributions: H.K.S. and S.B. designed research; H.K.S. and S.B. performed research; H.K.S. and S.B. analyzed data; H.K.S. and S.B. wrote the paper. 


\section{Introduction}

The orbitofrontal cortex (OFC), a part of the prefrontal cortex (PFC), is involved in flexible behavior (Miller and Cohen, 2001; Wallis, 2007; Fritz et al., 2010) by encoding specific stimulus-outcome or action-outcome expectancies as well as by dynamically revaluing such expectancies based on behavioral demands and motivational states (Delamater, 2007; Rudebeck et al., 2008; Wilson et al., 2014; Fresno et al., 2019). Specific OFC circuits can control specific aspects of flexible behavior and multiple reinforcement learning processes (Lee et al., 2012; Groman et al., 2019). For OFC neurons to encode the sensory attributes and subjective value of outcomes associated with external stimuli (Schoenbaum and Roesch, 2005; Delamater, 2007; Ostlund and Balleine, 2007), it requires sensory inputs to begin with. It is known that sensory stimulus-evoked signals in the OFC can distinguish between appetitive and aversive outcomes (Morrison and Salzman, 2011) associated with the stimuli. Further, the OFC can also influence sensory processing by modulating neuronal receptive fields in early sensory cortices, particularly the auditory cortex (AC; Winkowski et al., 2013).

To understand how specific stimulus outcome associations are created and how the stimulus-evoked OFC responses may influence sensory representation, it is crucial to delve into the origins of sensory inputs and sensory response properties of the OFC. In the case of auditory stimuli, the pathways involved and their contribution to auditory responses in the OFC are not known. What aspects of information in the ongoing auditory environment, how and in what form reaches the OFC would determine how mechanistically stimulus-outcome expectancies or values would get computed or updated. We, therefore, as a first step, consider the auditory evoked responses of OFC neurons, from a sensory perspective and attempt to decipher the crucial components of the auditory pathway involved in shaping auditory responses and their properties in the OFC.

Although mouse OFC single neurons have been shown to respond to a variety of sounds (Winkowski et al., 2018), it is unclear how selective the responses are and how they change under different sensory contexts. With single-unit recordings in mice, both awake and anesthetized, we show that auditory responses in the OFC are strongly context dependent with long timescale history dependence. We also show that the OFC neurons respond only to a change or deviant in the stimulus stream and cease to respond to any repeating stimuli from the first instant of repetition. We call this response characteristic as pure

This work was supported by the Wellcome Trust DBT India Alliance Grant IA/I/11/2500270, the Ministry of Human Resource Development (MHRD) Grant SSLS/VTA, the Indian Institute of Technology Kharagpur (IIT Kharagpur) Grant SGIGC-2015/DMN, and the Ministry of Human Resource Development Institute Fellowship.

Correspondence should be addressed to Sharba Bandyopadhyay at sharba@ece.iitkgp.ac.in.

https://doi.org/10.1523/ENEURO.0121-20.2020

Copyright (C) 2020 Srivastava and Bandyopadhyay

This is an open-access article distributed under the terms of the Creative Commons Attribution 4.0 International license, which permits unrestricted use, distribution and reproduction in any medium provided that the original work is properly attributed. oddball detection. We also show that these response properties are different from the AC, possibly arising either within the local circuits of the OFC or regions other than AC. Investigation of anatomic and functional sources of inputs show that both the lemniscal and non-lemniscal pathways at the cortical and subcortical levels shape auditory responses in the OFC. With pharmacological inactivation experiments, the contributions of multiple auditory cortical and subcortical areas in OFC's auditory responses were assessed. In the $A C$, the dorsal region (AuD), with the most projections to the OFC, surprisingly did not contribute to auditory responses of the OFC. In contrast, the other higher-order non-lemniscal ventral auditory area (AuV; Sacco and Sacchetti, 2010), was found to be the main source of auditory evoked excitatory drive to the OFC. The primary $A C(A 1)$ contributed to temporal response properties in the OFC. Further, considering the auditory thalamic (medial geniculate body; MGB) sources showed that nonlemniscal AuV's contribution to OFC responses originates from the lemniscal MGBv through its direct projections to AuV (Ohga et al., 2018). The non-lemniscal, polymodal medial division of MGB (MGBm; Weinberger, 2011; Lee, 2015) inactivation, however, caused the OFC auditory responses to become persistent, similar to frontal cortex responses during working memory dependent tasks (Fuster and Alexander, 1971; Funahashi et al., 1989; Schoenbaum and Setlow, 2001). Thus, the MGBm is a source that causes auditory driven long-lasting inhibition in the OFC. Inactivation of basolateral amygdala (BLA), providing inhibitory inputs (Dilgen et al., 2013; McGarry and Carter, 2016; Lichtenberg et al., 2017) to the OFC and known to receive MGBm inputs via the lateral amygdala (LA; Ledoux, 2000; Woodson et al., 2000), showed similar emergence of auditory driven persistent activity. Further, the same pathway contributed substantially to the strength of deviant selectivity in the OFC. We suggest that the feedforward inhibition (Dilgen et al., 2013) from BLA to OFC and parallel MGBm to LA inhibition (Woodson et al., 2000) transmitted to OFC via BLA allow for two independent controls to generate persistent activity in the OFC required for stimulus outcome associations.

\section{Materials and Methods}

\section{Animals}

All animal experiments were approved by Institutional Animal Ethics Committee (IAEC) of Indian Institute of Technology Kharagpur. Animals were reared under a 12/12 h light/dark cycle and maintained at a temperature of 22$25^{\circ} \mathrm{C}$ and had access to food and water ad libitum. C57BL/6 mice of either sex, postnatal $(P)$ age between $\mathrm{P}-25$ and $\mathrm{P}$ 45 , were used for the experiments (Table 1). Data acquired in pharmacological block experiments before blocking were also included in the analysis of OFC responses.

\section{Animal preparation \\ Anesthetized recordings}

Animals were anesthetized using isoflurane (5\% for induction and around 1-1.5\% for maintenance). Body temperature was maintained at $39^{\circ} \mathrm{C}$ by placing the animal on a heating plate. A small incision was made to expose the 
Table 1: Number of animals used by experiment

\begin{tabular}{ll}
\hline Experiment & Number of mice \\
\hline OFC electrophysiology & 27 \\
AC electrophysiology & 5 \\
Anatomy & 9 \\
A1 inactivation (+ bilateral) & $7(+2)$ \\
AuV inactivation & 5 \\
AuD inactivation & 3 \\
MGBv inactivation & 2 \\
MGBm Inactivation & 5 \\
BLA inactivation & 5 \\
Awake OFC electrophysiology & 5 \\
Total & 74 \\
\hline
\end{tabular}

skull and a metal plate was attached on to the skull to head fix the animal. Once head fixed, a small $(\sim 2 \mathrm{~mm}$ in diameter) craniotomy was performed to remove the skull over the recording site. For OFC, the stereotaxic coordinates used were $A P=+2.5 \mathrm{~mm}, \mathrm{ML}=1 \mathrm{~mm}$ from the bregma, DV $=1.8 \mathrm{~mm}$ (Paxinos and Franklin, 2013) from the brain surface. For AC, the recording site was identified based on the vasculature (Sawatari et al., 2011). All recordings were performed with a microelectrode array (MEA) with a $4 \times 4$ grid ( $125 \mu \mathrm{m}$ between rows and columns) of epoxycoated tungsten electrodes (MicroProbes, impedance $\sim 3-5$ $\mathrm{M} \Omega$ ).

\section{Awake head-fixed recordings}

As in the anesthetized case, a similar but smaller craniotomy ( $\sim 1 \mathrm{~mm}$ in diameter) was performed and the electrodes were advanced into the recording site and the held fixed on the skull using Metabond (C \& B superbond). A titanium plate was also fixed to the skull (posterior to the electrodes) with Metabond to head-fix the animal during the experiments. Animals were allowed to recover for $5 \mathrm{~d}$ and then were habituated with the recording setup for $30 \mathrm{~min}$ for $3 \mathrm{~d}$ before data collection. Data collection lasted for $<7 \mathrm{~d}$, with $\sim 1$-h-long sessions every day. Units collected on each day from the recording electrodes were considered as separate units.

\section{Stimulus}

All acoustic stimulation was presented from the right side, contralateral to the recording site. Initially, noise bursts [6- to $48-\mathrm{kHz}$ bandwidth, $50 \mathrm{~ms}$, 5 -s intertrial interval (ITI), of multiple intensities, 40- to $0-\mathrm{dB}$ attenuation, in $10-\mathrm{dB}$ steps; $0-\mathrm{dB}$ attenuation corresponds to $\sim 90-\mathrm{dB}$ SPL for tones] were used to obtain threshold sound level for noise. Next single units were characterized with pure tones $(50 \mathrm{~ms}, 6-48 \mathrm{kHz}, 1 / 2$ octave apart, $70-$ to $80-\mathrm{dB}$ SPL, depending on noise threshold, $5 \mathrm{~s}$ apart, except mentioned otherwise) to obtain tuning and the best frequencies (BFs) at the chosen sound level. Next response to a pair of oddball stimulus set was collected. The oddball stimulus consisted of a standard token $(\mathrm{S} ; 50 \mathrm{~ms}$, either a noise token or a pure tone) and deviant token (D; $50 \mathrm{~ms}$, either a pure tone or a noise token, respectively, for noise-tone, NT or TN, oddball; S and D were both pure tones in case of tone-tone, $T \mathrm{~T}$, oddball). The S-D stream had 15 tokens usually presented at $4 \mathrm{~Hz}$ or $3.3 \mathrm{~Hz}$; all the tokens were $S$ tokens except the eighth token which was the $\mathrm{D}$ token. In the second of the pair of the oddball stimulus set, the $S$ and $D$ tokens were swapped. Each oddball set was repeated 20-30 times with a gap of $>5 \mathrm{~s}$ between each repetition. In some animals, the total number of tokens was changed to 20 , and the location of deviant was also changed to 12th. In these animals, the response profile was not different from the usual 15 tokens case. All sound tokens presented in all kinds of stimuli had 5-ms rise and fall times. Since recordings were with MEAs with 16 electrodes, the pure tone frequency was chosen based on the tuning of the majority of simultaneously recorded neurons, such that the chosen tone frequency was within the receptive field of most neurons. The stimuli were generated using custom-written software in MATLAB (MathWorks) and presented with Tucker Davis Technologies (TDT) ES1 speakers (driven with TDT ED1 drivers) after generation with a TDT RX6 processor and attenuated using a TDT PA5. The speaker was placed $10 \mathrm{~cm}$ away from the contralateral ear.

\section{Electrophysiology \\ Anesthetized}

The MEA was slowly advanced into the recording site with the help of a manipulator (MP-225, Sutter). The electrodes were allowed to settle and stabilize for $\sim 30 \mathrm{~min}$ before the data were acquired. Data were collected using custom-written software (MATLAB), through a unity gain headstage (16 channels, Plexon HST 16025) amplifier, followed by a preamplifer (PB3, Plexon, 1000×). Wide-band neural signals $(0.7 \mathrm{~Hz}$ to $8 \mathrm{kHz})$ as well as a parallel set of 16 channels with spike signals $(150 \mathrm{~Hz}$ to $8 \mathrm{kHz})$ were stored after digitizing at $20 \mathrm{kHz}$ using a A/D board (National Instruments). Off-line analysis was performed with stored data. At the end of the experiment, the animal's brain was harvested for post hoc examination of the recording site.

\section{Awake}

For awake recordings, the animal was placed in a small tube with head protruding out and fixed using the titanium plate implanted during surgery. The rest of the procedure was similar to the anesthetized recordings.

\section{Anatomy}

Nine animals were injected with $200 \mathrm{nl}$ of green retrobeads (Lumafluor) into OFC and three of them were also injected with $100 \mathrm{nl}$ of anterograde tracing AAV.CB7.Cl. $m$ Cherry in MGBv using Nanoject II. After $14 \mathrm{~d}$ of injection, the animals were transcardially perfused with 20-ml PBS followed by $20 \mathrm{ml}$ of $4 \%$ paraformaldehyde and brain was harvested and kept in 4\% paraformaldehyde overnight. $100-\mu \mathrm{m}$-thick brain sections were cut using a vibratome (Leica VT1000S), mounted on a glass slide with fluomount cover slip, and observed under a fluorescence microscope (Leica DM2500).

\section{Electrophysiology with pharmacological inactivation}

A small burr hole was made on the skull over the area to be inactivated, ipsilateral (or both sides as mentioned) to the recording site. A Hamilton syringe (7000 series) loaded with the $200 \mathrm{nl}$ of GABA agonists $(5 \mu \mathrm{g} / \mu \mathrm{l}$ muscimol and 
$2 \mu \mathrm{g} / \mu \mathrm{l}$ baclofen) or equal volume saline was inserted and held via a cannula implanted on the skull with dental cement. Only after the syringe was positioned securely, the electrodes were inserted into the recording site as described above. For injecting the agonists/saline during the experiment, the Hamilton syringe was gently pressed/ tapped multiple times over a 5- to 10-s period to release the entire volume. There was a waiting period of $30 \mathrm{~min}$ for the agonists to have their effect before the next data set was acquired. SR101 was added to the mixture of agonists or saline for post hoc confirmation of the target site and spread of the injection. Different divisions of $A C$ were identified and marked based on the vasculature. For MGB (Slater et al., 2019) and BLA (Luna and Morozov, 2012) following stereotaxic coordinates were used: MGB: AP = $-3.27 \mathrm{~mm}, \mathrm{ML}=2.0 \mathrm{~mm}$ from bregma, $-3.0 \mathrm{~mm}$ from the brain surface; and BLA: AP $=-1.3 \mathrm{~mm}, \mathrm{ML}=3.2 \mathrm{~mm}$ from bregma, 3.8 from the brain surface.

\section{Data analysis}

Spike Sorting was done offline in custom-written MATLAB software. Data were notch filtered (Butterworth fourth order) to reject any remnant power supply $50-\mathrm{Hz}$ oscillations. Spiking activity was obtained directly from the spike channels of the PBX3 preamp. Waveform fluctuations above 3.5-4 SDs (usually 4) from the baseline were isolated and based on shapes, spike waveforms were clustered into different groups. The timing of spikes with respect to data collection onset (and hence also stimulus presentation) was extracted for each spike shape (single unit) for further analysis. A single unit was considered as responsive if the spike rate within $400 \mathrm{~ms}$ ( $200 \mathrm{~ms}$ in case of awake condition) of stimulus presentation was significantly different from the baseline $(300 \mathrm{~ms}$ preceding the stimulus, $t$ test, $\alpha=0.05$ ). Response latency was calculated as the time at which the spike rate in the average peristimulus time histogram (PSTH; 20-ms bins) was maximum.

\section{Narrow tuning and calculation of $B F$}

Tuning of neurons was considered to be narrow (well tuned) or bimodal and/or broad. The frequency corresponding to the maximum response rate out of the seven frequencies presented to narrowly-tuned units (below) was considered the BF of the unit. Those units were narrow -tuned whose average response to frequencies other than one octave around BF was 2 SDs (variability in response rate at $\mathrm{BF}$ ) below the response at $\mathrm{BF}$.

\section{Spatial BF variability}

The SD of BFs of all the simultaneously acquired units (only narrowly tuned, above) was normalized by the product of area accommodating these responding units and the number of units:

$$
=\frac{\text { Spatial BF variability }(B F s \text { of simultneously acquired units })}{\text { area } X \text { number of units }} .
$$

For simulating a distribution of completely heterogeneous BFs, each unit was randomly assigned a BF (uniformly over the seven frequencies used) and BF variability was calculated with 1000 bootstraps.

\section{CSI calculation}

For common selectivity index (CSI) calculation, those units were included which responded to at least one of the four stimulus tokens, first of each of the $S$ tokens in the oddball pair $S_{X}$ and $S_{X S}$ (XS being the swap of the X oddball), and the deviant tokens, $D_{x}$ and $D_{x s}$. CSI was calculated as per the following equation (Ulanovsky et al., 2003):

$$
C S I=\frac{\left(D_{X}+D_{X S}\right)-\left(S_{X}+S_{X S}\right)}{\left(D_{X}+D_{X S}\right)+\left(S_{X}+S_{X S}\right)}
$$

where $S_{i} / D_{X / X S}$ represents the mean rate response to those tokens, $S_{i}$ the $i^{\text {th }}$ token and $S_{P T}$ being the token preceding the $D$ token. The rate responses in each case were computed based on the following windows: for $S_{1}, 100$ $400 \mathrm{~ms}$ from $\mathrm{S}_{1}$; for $\mathrm{S}_{\mathrm{ALL}}, \mathrm{S}_{2}+100 \mathrm{~ms}$, until deviant, entire length; for $S_{\mathrm{PT}}, 100 \mathrm{~ms}$ from $\mathrm{S}_{\mathrm{PT}}$ up to deviant; and for $\mathrm{D}$, $100-400 \mathrm{~ms}$ from deviant.

When a sufficient sized population (at least 50 units) of paired data were not available (as in the case of tone-tone, TT oddball and in before versus after pharmacological inactivation experiments), deviant selectivity index (DSI) of each unit was calculated as follows:

$$
D S I=\frac{D_{x}-S_{X}}{D_{X}+S_{X}}
$$

\section{Anatomy}

The number of retrobeads was quantified using a threshold that was manually set for individual sections depending on the background intensity. The laminar demarcation was based on distance from pia, which was also corroborated in a subset with the MGBv projections observed with AAV.CB7.Cl.mCherry. All beads within $300 \mu \mathrm{m}$ from the pia were marked as layer 2-3, beads between 300 and $450 \mu \mathrm{m}$ (or mCherry) were marked as layer four and beads below that were grouped into layer 5-6.

Demarcation of AuV, A1, and AuD to confirm correct injections through the spread of SR101 in blocking solution was through brain atlas (Paxinos and Franklin, 2013) and distance from rhinal fissure. Only those animals were included in the dataset whose spread was post hoc confirmed as above. Injections in MGBv and MGBm were targeted and post hoc confirmed through brain atlas (Paxinos and Franklin, 2013).

\section{Response duration}

A sliding response window of size $100 \mathrm{~ms}$ starting from the stimulus start, in 20-ms steps, was compared with the random 100-ms window in the baseline for significance. Consecutive significant bins with a time difference of $<100 \mathrm{~ms}$ between them were joined together for the determination of response duration. 


\section{Spike timing jitter}

The spike timing jitter expressed as variability in the timing of individual spikes across trials was computed as the reliability $\left(R_{\text {corr }}\right)$ in spike timing which is a measure of similarity between pairs of individual spike trains (Schreiber et al., 2003). The trial wise individual spike trains binned at $1 \mathrm{~ms}$ was convolved with a Gaussian filter of $\sigma=2 \mathrm{~ms}$, and then the coefficient of correlation was calculated between all pairs of trials using the MATLAB function corrcoef. Higher the $\mathrm{R}_{\text {corr }}$ value, less is the spike timing jitter.

\section{Pairwise correlations}

Pairwise correlations were calculated as correlation coefficient using the MATLAB function corrcoef between mean PSTHs (from stimulus start) of simultaneously recorded units.

\section{Results}

\section{OFC neurons respond to sound with very long timescale dependence}

To first obtain OFC single neuron responses to sounds, independent of behavioral state, active memory, and other cognitive processes, we recorded single units in the anesthetized mice. The recording sites were confirmed to be in the OFC (lateral and/or ventral) by post hoc Nissl staining of coronal sections (Fig. 1A). We found that neurons in the mouse OFC robustly responded to sound stimulation (Fig. 1B,C) with broadband noise and pure tones (also see Winkowski et al., 2018), with typically monotonic rate intensity functions with noise (Fig. $1 B$, right) and variety of BFs (Fig. $1 \mathrm{Ci}$-Civ) with narrow (Materials and Methods; $45 \%$ of the responding units) and bimodal to broad tuning $(55 \%$ of the responding units; Fig. $1 \mathrm{Cv}$, Cvi). While most of the responding units increased their firing rate at $\mathrm{BF}(\sim 86 \%, 816 / 949$ units), about $\sim 14 \%$ (133/949) of units reduced their firing rate at some frequency (Fig. 1D); 76 of the 133 units were excited at BF while the rest showed no excitatory responses. Notably, the suppression observed in all the 133 units (Fig. 1D), were all at either 6 or $8.5 \mathrm{kHz}$. The mean peak latency observed at the BF of units with excitatory responses was $284 \pm 3 \mathrm{~ms}$ (Fig. 1E). The latency was substantially longer than what is seen in the mouse AC single-unit responses but similar to the late component of auditory responses (Chen et al., 2015), likely in the non-A1.

To check for the possible topographical organization of tuning based on the narrowly tuned units in the horizontal plane of the OFC, we considered the variability of BF of simultaneously recorded units. The distribution of calculated spatial BF variability in simultaneously recorded units (Eq. 1; Materials and Methods) across all recording locations was compared with a distribution expected with completely random BFs at each recording site (Materials and Methods; Fig. 1F, black arrow median of data, gray arrow median of the distribution with spatially random BFs). The two distributions were not significantly different (Kolmogorov-Smirnov test, $p=0.06)$. This suggests that the local organization is entirely random, indicating the absence of any BF based organization at the spatial scales $(\sim 400 \mu \mathrm{m})$ of our recording along the horizontal plane in OFC. Distribution of level thresholds for broadband noise is shown in Figure 1G. The noise (6- to 48-kHz bandwidth) threshold in $\mathrm{dB}$ attenuation of our system corresponds to $\sim 20 \mathrm{~dB}$ above tone threshold. Thus, tones used in the study were played usually at an attenuation that is $10 \mathrm{~dB}$ above or at the noise threshold, which corresponds to $20-30 \mathrm{~dB}$ above the usual tone threshold in the 6- to $48-\mathrm{kHz}$ range.

To investigate the effects of adaptation and stimulus history, we recorded OFC responses to pure tone presentation with varying ITI with a short interval (1 and $3 \mathrm{~s}$ ) less than $5 \mathrm{~s}$, mid-interval ranging from 5 to $7 \mathrm{~s}$ and long interval ranging from more than 7 up to $11 \mathrm{~s}$ (Fig. $2 A, C)$ and compared it to AC (Fig. 2B,F, both $A 1$ and AuV; determined from post hoc Nissl stains with electrode tracks). We found that the response profiles of these three ITIs in the OFC were significantly different with one another (one-way ANOVA; Fig. 2C,D). The peak spike rate of the long ITI group was significantly different (one-way ANOVA, $p<0.001$ ) from the mid and short ITIs (Fig. 2D), whereas the latency (Fig. $2 E$ ) of the short group was significantly different from the other two groups (one-way ANOVA $p<0.01$ between short and long and $p<0.001$ between short and mid). These results indicate that OFC neurons show very long (at least up to tens of seconds) timescales of stimulus history dependence that is reflected in the spike rate or latency of the response. Similar analyses of the AC neurons (Fig. $2 F-H$ ) show that neither the peak spike rates (Fig. $2 F, G$ ) nor the response latencies (Fig. $2 H$ ) were different in the three groups. Thus, OFC responses to auditory stimuli were found to have unprecedented temporal stimulus history dependence. Such remarkable dependence of sensory responses on long stimulus history, unlike in the sensory cortex, would be crucial in normal environments with continuously varying sensory inputs.

\section{Neurons in the OFC show high context dependence and pure oddball detection unlike in the AC}

Given the long temporal dependencies, presumably because of strong and long-lasting adaptation, it becomes important to find the key aspects in streams of sounds to which neurons in the OFC respond. Since neurons in the OFC responded to both tones and noise (Fig. 1), we collected OFC responses to an oddball stimulus set with noise tokens as the standard (S) stimulus with a pure tone embedded in the stream as the D token (NT) and its swap (TN; Fig. 3A; Materials and Methods). We found that OFC neurons robustly responded to the $D$ token (Fig. 3B). Typically, a strong onset response to the first of the standards (a deviant/change from the pre-stimulus silence) was seen, followed by a strong response to the $D$. This pure oddball detection characterized by responses to the first $S$ token $\left(S_{1}\right)$ and $D$, followed by strong adaptation such that there is no response to the succeeding tokens, is not seen in AC. Thus, the responses in the OFC to oddball stimulation show strong and fast stimulus-specific adaptation (Taaseh et al., 2011; Nieto-Diego and Malmierca, 2016). 
A
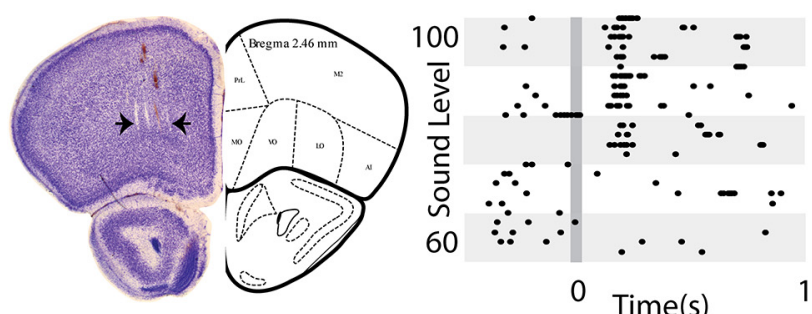

B
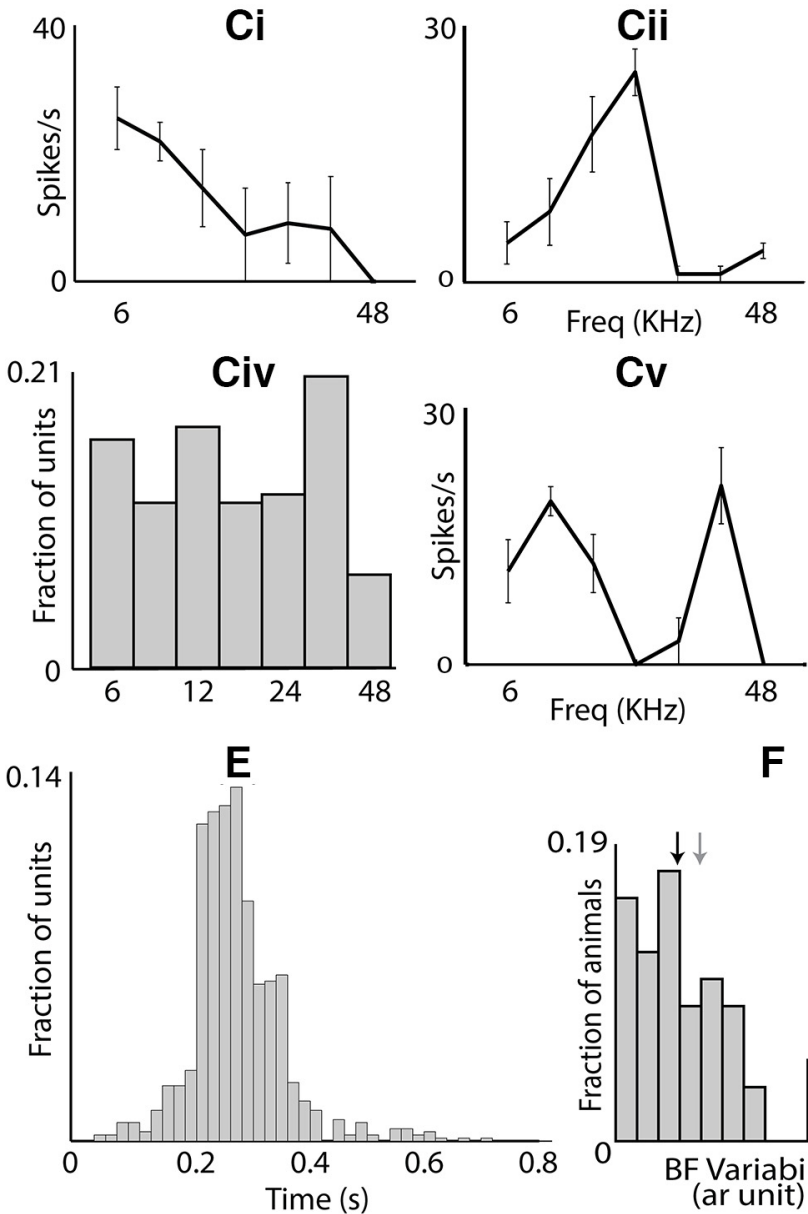

Cv

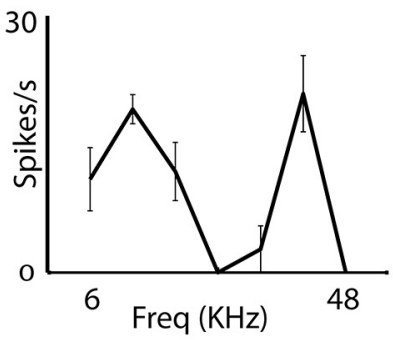

$\mathbf{F}$

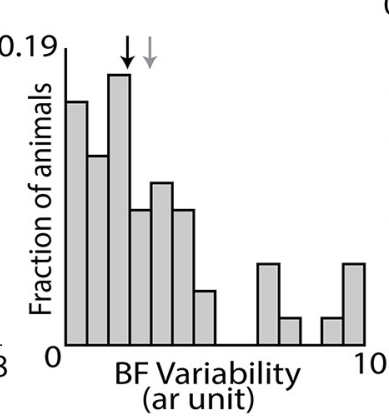

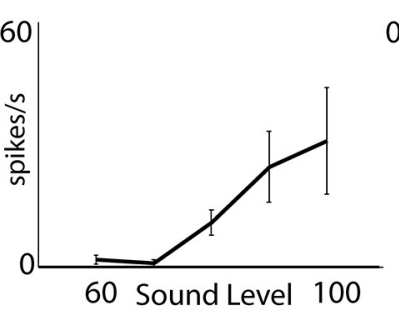
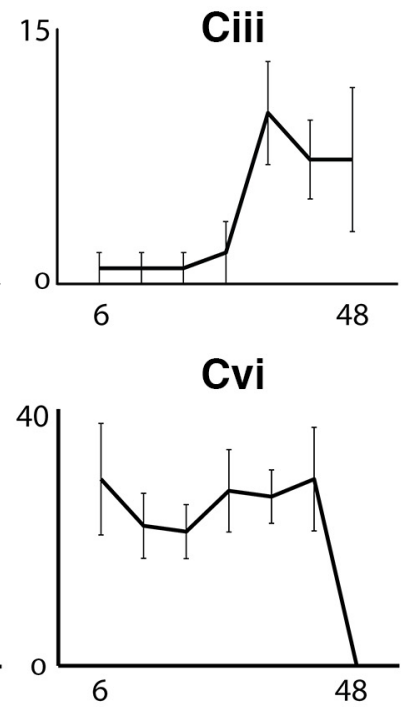

G

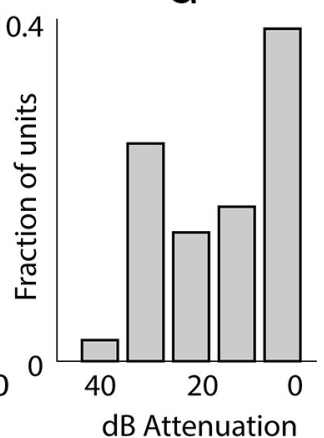

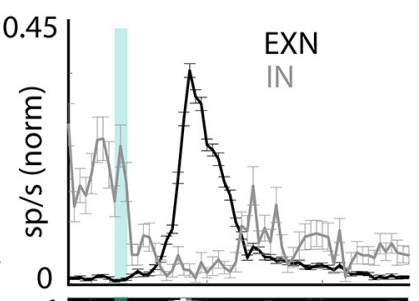

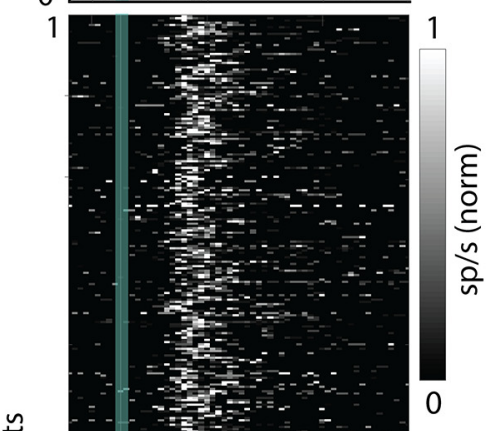

Figure 1. OFC responds to auditory stimulation. $\boldsymbol{A}$, Nissl stain of OFC section showing electrode tracks. Black arrows mark the medio-lateral extent of the electrode array. $\boldsymbol{B}$, An example unit showing responses to increasing intensities of broadband noise in raster plot (left) and rate-level curve (right). $\boldsymbol{C i}$-Ciii, Tuning curves of three example units tuned to low (Ci), mid (Cii), and high (Ciii) frequencies. Civ, Distribution of units tuned to different frequencies. $\mathbf{C v}$, An example unit with bimodal tuning curve. $\mathbf{C v i}$, An example unit with broad tuning curve. $\boldsymbol{D}$, top, Mean normalized PSTH \pm SEM of all units showing excitation (black) and inhibition (gray) on auditory stimulation. Bottom, Individual unit's PSTH (upper panel: units showing excitation, middle two panels: units showing excitation to some frequency and inhibition to some other frequency, lower panel: units showing inhibition). $\boldsymbol{E}$, Distribution of peak response latency to the BF. $\boldsymbol{F}$, Spatial BF variability. Black arrow marks the median of the distribution and gray arrow marks the median of the distribution of completely random BF organization. $\mathbf{G}$, Distribution of broad band noise thresholds; 0-dB attenuation corresponds to $\sim 90-\mathrm{dB}$ SPL for a tone at $17 \mathrm{kHz}$.

We obtained responses to oddball stimulus pairs (NT and TN) from 109 OFC units (10 animals, 218 cases; Table 2 ), where a unit was considered responding if it responded to at least one of the four stimulus tokens $\left(S_{1}\right.$ and $D$ in each of the two in a pair; Materials and Methods). In $36 \%$ of the cases, units responded to both the $S_{1}$ and the $D$ token (Fig. $3 C$, black; Table 2), while in $31 \%$ cases, units responded to $S_{1}$ but not $D$ (Fig. $3 C$, magenta; Table 2). Further, $17 \%$ responded to only the $D$ token (Fig. $3 C$, blue; Table 2) and the remaining $16 \%$ did not respond to either. In the last $16 \%$ cases, there was however a response to one of the stimulus tokens $\left(S_{1}\right.$ and/or $\left.D\right)$ in the corresponding swap oddball stimulus. Thus, although OFC neurons were found to be generally responsive to 


\section{OFC}

A
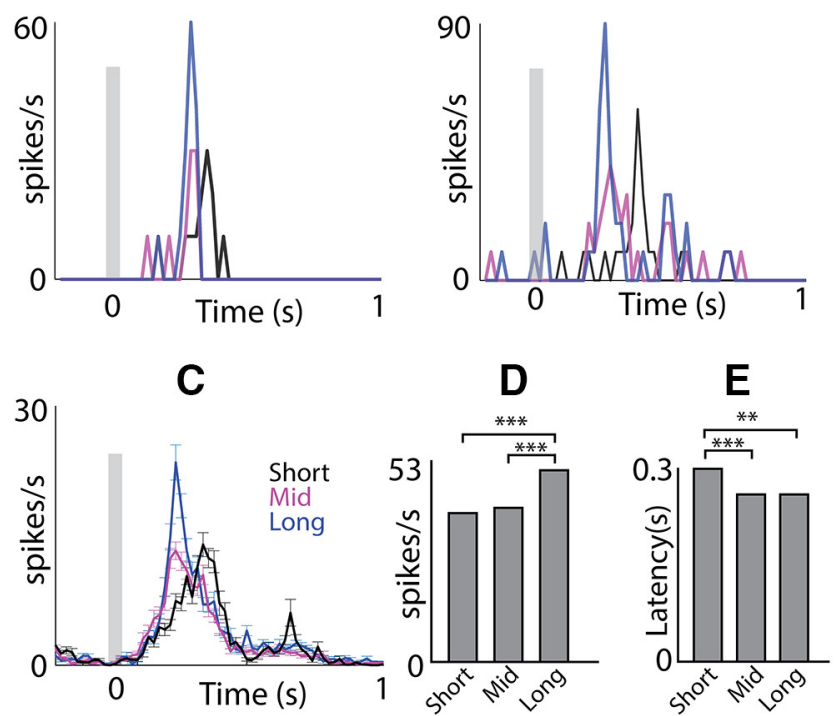

AC

$B$

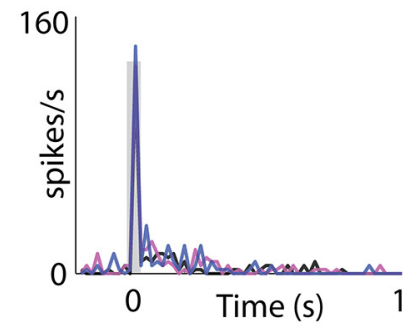

F

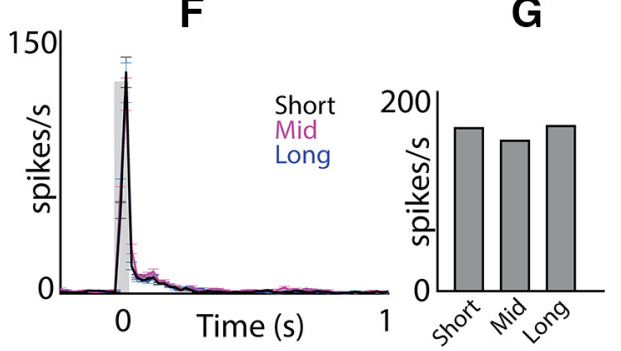

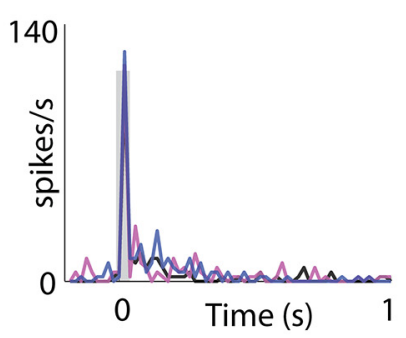

G

H

Figure 2. OFC neurons show long timescales of adaptation. $\boldsymbol{A}$, Psth of two example units in the OFC at different ITls [black; $<5 \mathrm{~s}$ (short), magenta; between 5 and $7 \mathrm{~s}$ (mid), blue; $>7 \mathrm{~s}$ up to $11 \mathrm{~s}$ (long)]. $\boldsymbol{B}$, Similar to $\boldsymbol{A}$ in the AC. $\boldsymbol{C}$, Mean population PSTH \pm SEM at different ITIs in the OFC. $\boldsymbol{D}$, Mean peak spike rate in the OFC. $\boldsymbol{E}$, Mean peak response latency in the OFC. $\boldsymbol{F}$, Mean population $\mathrm{PSTH} \pm \mathrm{SEM}$ at different ITIs in the AC. $\boldsymbol{G}$, Mean peak spike rate in the AC. $\boldsymbol{H}$, Mean peak response latency in the $A C .{ }^{* \star} p<0.01$, ${ }^{* \star *} p<0.001$.

both, the tone and the noise when played as an individual token (Fig. 1), their response to the same sound token changed depending on the context of the stimuli (as in oddball stimulus). We find that when a neuron responded to a tone as $S_{1}$ (Table 2), it was less likely to respond to the noise token as D (65/82: did not respond to noise as D and 17/82: responded to noise as $D, p<0.001$, two proportion $z$ test; Table 2). On the contrary, if a neuron responded to the noise token as $\mathrm{S}_{1}$ (Table 2), it was more likely to also respond to the tone token as $D(62 / 65$ : responded to tone as $D$ and 3/65: did not respond to tone as $D, p<0.001$, two proportion $z$ test; Table 2). Such context-specific selectivity is contrary to expectations, as a broadband noise as $S$ is likely to adapt all frequency channels, thus masking the response to a tone as D embedded in a sequence of noise tokens. The above suggests that the OFC auditory circuitry is inherently more selective in detecting narrowband sounds in broadband background noise. However, when considering the response of the same unit to a stimulus token (noise or tone) as the $S$ and as $D$, we find that out of 109 units, 75(17), 7(48), 8(16), and 19(28) units responded to the tone (noise) both as $S$ and $D$, only as $S$, only as $D$, and neither, respectively (Table 3 ). The above suggests that responses to the tone were less context-sensitive than noise with more units responding to tones than to noise (tones: $75 / 109$ and noise: $17 /$ 109, $p<0.001$, two proportion $z$ test) independent of their occurrence as $S$ or $D$. This further corroborates the fact that the OFC neurons inherently are more responsive to tones and hence likely to narrowband tonal sounds like mouse vocalizations.
In contrast, we found nearly an opposite pattern of context dependence in the AC. Responses to oddball stimuli pairs (NT and TN) from a total of 62 units (51 in A1, 11 in AuV, 124 cases; Fig. 3D) were collected from the $A C$ that responded to at least one of the four stimulus tokens (as above). In $22 \%$ of the cases, units responded to both $\mathrm{S}_{1}$ and D (Fig. 3D, black, bar plot; Table 4), while in $48 \%$ cases, units responded to $\mathrm{S}_{1}$ but not $\mathrm{D}$ (Fig. 3D, magenta, bar plot; Table 4). Further, $20 \%$ responded to only the $D$ token (Fig. 3D, blue, bar plot; Table 4), and the remaining $10 \%$ did not respond to either. As expected from adaptation along frequency channels, when there was a response to the noise as $\mathrm{S}$, very few units responded to the tone as $D(7 / 61)$ and most were unresponsive to the tone (54/61), opposite of what was observed in the OFC. Considering responses of the same neuron to the tone or noise token in either of the two contexts (as $S_{1}$ or D), 7 (45), 20(16), $0(1)$, and 35(0) out of $62 \mathrm{AC}$ units responded to tone (noise) as both $S_{1}$ and $D$, only as $S_{1}$, only as $D$ and neither of the two, respectively (Table 5). In the AC, contrary to observations in the OFC, the tone responses independent of $S$ and $D$ were absent, while noise responses occurred almost independent of $S$ and $D$ (tones: 7/62 and noise: $45 / 62, p<0.001$, two proportion $z$ test). There were many more cases in the AC when there were no responses to the tone either as $S$ or as D. Such units were present in both $\mathrm{A} 1$ and $\mathrm{AuV}$ in similar proportions (30/51 and $5 / 11$, NS). Although the choice of the tone frequency (Tf) for oddball sets (NT and TN) with respect to the BF of neurons were similar for OFC and AC (mean(|BF-Tf|) 1.3 octaves for OFC and 1.1 octaves for AC, not significant (NS), unpaired $t$ test), the more number cases where there 
A
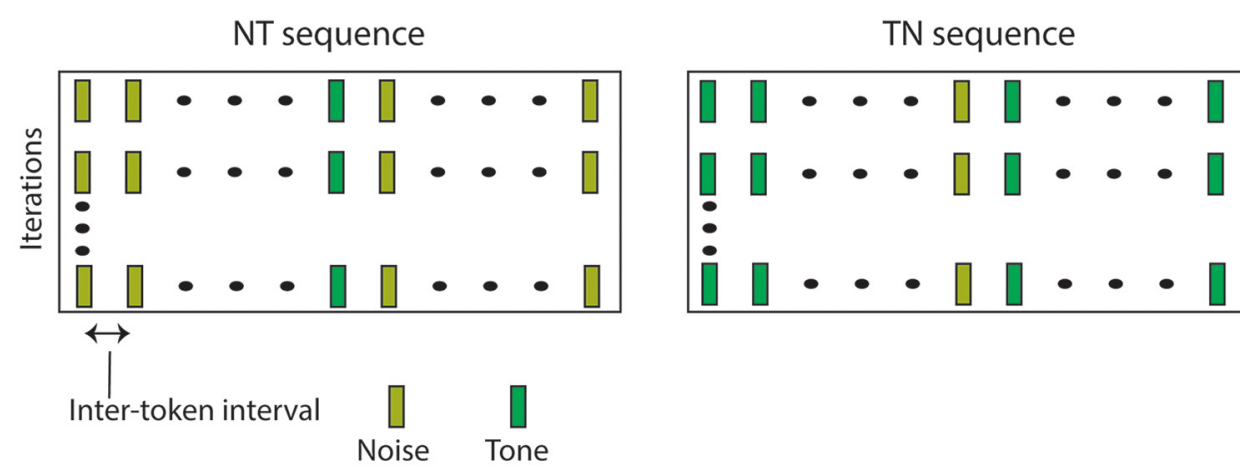

B
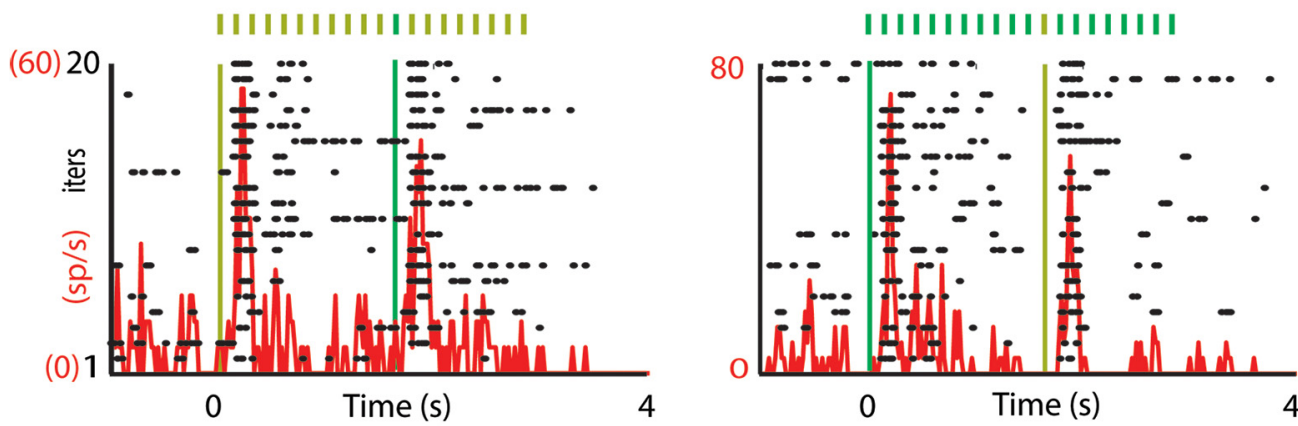

C
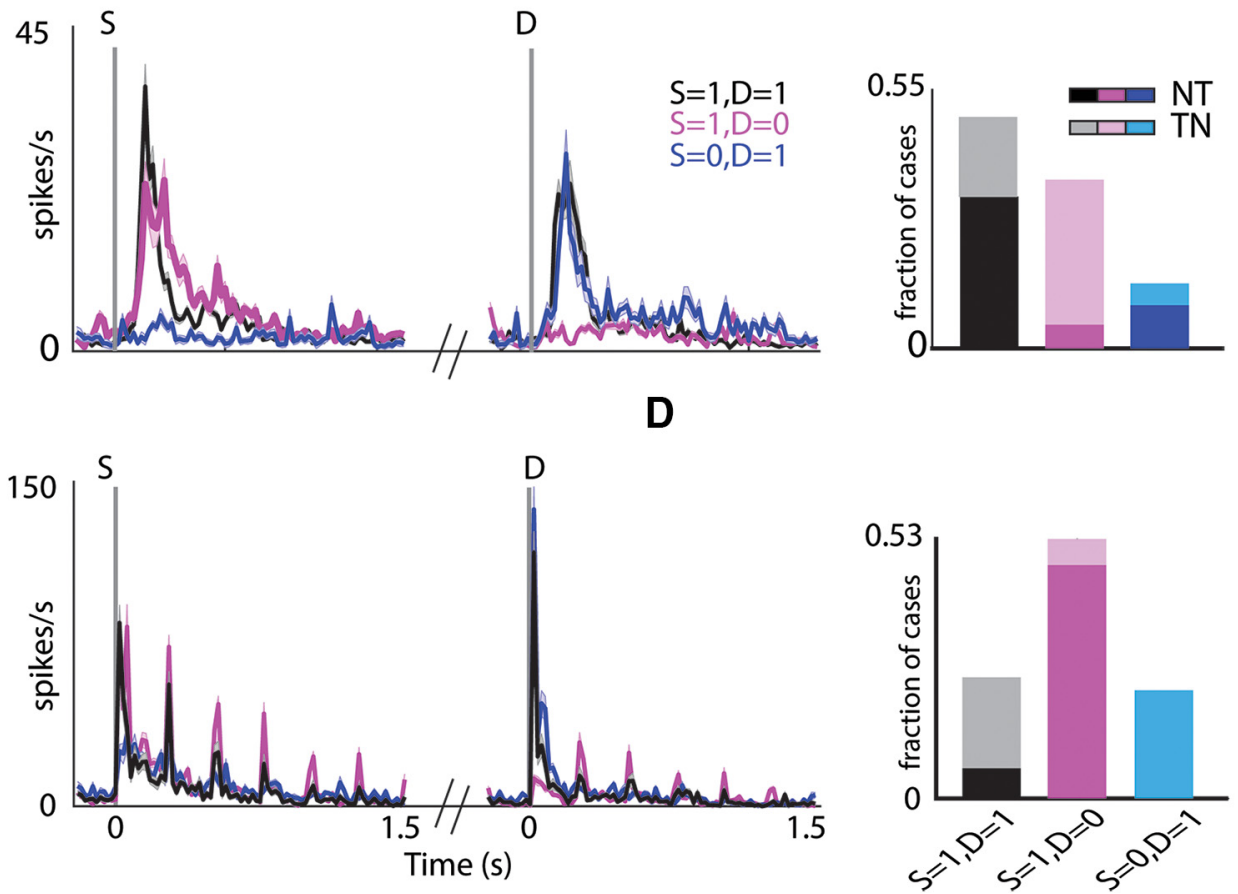

Figure 3. Pure oddball Detection in OFC unlike AC (i). $\boldsymbol{A}$, Oddball Stimuli showing NT (left) and TN (right) sequences. $\boldsymbol{B}$, An example unit showing auditory response (raster (black) and PSTH (red) to an oddball stimulus where noise is played as standard and tone as deviant (NT; left) and its swap (TN; right). The vertical lines mark the onset of standard and deviant (yellow for noise and green for tone). $\boldsymbol{C}$, left, Mean population PSTH \pm SEM of units responding to both standard onset and deviant $(S=1, D=1$; black), responding only to standard onset $(S=1, D=0$; magenta) and responding only to deviant $(S=0, D=1$; blue). Right, Fraction of units belonging to different categories as described in $\boldsymbol{B}$. The darker shades show the fraction of units belonging to NT and lighter shades show fraction of units belonging to TN group. $\boldsymbol{D}$, Similar to $\boldsymbol{C}$ in the AC. 
Table 2: Different response types to NT and TN stimuli in the OFC

\begin{tabular}{llll}
\hline & $\mathrm{S}_{1}=1$ and $\mathrm{D}=1$ & $\mathrm{~S}_{1}=1$ and $\mathrm{D}=0$ & $\mathrm{~S}_{1}=0$ and $\mathrm{D}=1$ \\
cases & cases \\
218 cases & cases & 03 & 21 \\
\hline NT & 62 & 65 & 16 \\
TN & 17 & $68(31 \%)$ & $37(17 \%)$ \\
Total & $79(36 \%)$ & & \\
\hline
\end{tabular}

was no response to tones (as $\mathrm{S}$ or $\mathrm{D}$ ) in $\mathrm{AC}$ could be because of narrower tuning profiles. To test this, we checked whether the units that did not respond to tones in the oddball stimulus responded to individual tone pip or not. We found that $27 \%(17 / 62)$ responded to both the stimuli, whereas $23 \%(14 / 62)$ units showed contextual modulation with 10 units responding only to oddball stimulus and 4 units responding only to tone pip. Remaining $50 \%$ of the units $(31 / 62)$ did not respond in both the cases reflecting narrow tuning profile in the $\mathrm{AC}$. This further corroborates with the finding that OFC neurons are more broadly tuned (Fig. 1) as compared with AC.

To find the neuron's inherent preference to the deviant either T or N, we calculated CSI in three different ways based on the choice of $S$ tokens considered for $S_{X}$ and $S_{X S}$ in Equation 2. We compared the spike rates in response to either the $S_{1}$ and $D$ or all standard tokens $\left(S_{A L L}\right)$ preceding $D$ except $S_{1}$ or just the previous token $\left(S_{P}\right)$ to $D$. Comparison of responses in scatter plots (Fig. 4A) of spikes rates of $S_{1}$ and $D$ (left), $S_{A L L}$ and $D$ (middle) and $S_{P}$ and D (right) for NT and TN (blue and orange, respectively) shows lack of responses to $\mathrm{S}$ beyond $\mathrm{S}_{1}$. Further, the different CSI distributions (Fig. $4 A$, bottom) show that the OFC neurons respond strongly to $S_{1}$ as compared with $D$ probably because $S_{1}$ is a bigger change than $D$ in the stimulus space. The scatter plots of spike rates and CSI indices of $S_{A L L}$ and $D$, and $S_{P}$ and $D$ look very similar, suggesting that OFC neurons do not respond to any $S$ except $S_{1}$. The CSI distributions calculated by taking either $S_{A L L}$ and $D$ or $S_{P}$ and $D$, were not significantly different in the OFC, whereas in $A C$ these two distributions were significantly different $(p<0.001, t$ test; Fig. $4 B)$. These results indicate a strong adaptation onset right from the second token (beginning of repetition) in OFC, unlike AC suggesting a hierarchy of SSA strength along this pathway.

One of the possible reasons that OFC neurons do not respond to any of the $S$ tokens following the response to $\mathrm{S}_{1}$ could be faster rate of stimulus presentation. So instead of the 200 (usually)/250 ms intertoken interval (ITol), we also considered ITols of 300 (27 units), 400 (41 units),

Table 3: OFC response types depending on the stimulus type and its location in the sequence

\begin{tabular}{lllll}
\hline & $\begin{array}{l}\mathrm{S}_{1}=1 \text { and } \\
\mathrm{D}=1 \\
\text { (units) }\end{array}$ & $\begin{array}{l}\mathrm{S}_{1}=1 \text { and } \\
\mathrm{D}=0 \\
\text { (units) }\end{array}$ & $\begin{array}{l}\mathrm{S}_{1}=0 \text { and } \\
\mathrm{D}=1 \\
\text { (units) }\end{array}$ & $\begin{array}{l}\mathrm{S}_{1}=0 \text { and } \\
\mathrm{D}=0 \\
\text { (units) }\end{array}$ \\
\hline $\begin{array}{c}\text { Tone anits } \mathrm{S}_{1} \\
\text { or D }\end{array}$ & 75 & 07 & 08 & 19 \\
$\begin{array}{c}\text { Noise as } \mathrm{S}_{1} \\
\text { or D }\end{array}$ & 17 & 48 & 16 & 28 \\
& & & &
\end{tabular}

Table 4: Different responses types to NT and TN stimuli in the AC

\begin{tabular}{llll}
\hline & $\mathrm{S}_{1}=1$ and $\mathrm{D}=1$ & $\mathrm{~S}_{1}=1$ and $\mathrm{D}=0$ & $\mathrm{~S}_{1}=0$ and $\mathrm{D}=1$ \\
cases & cases \\
124 cases & cases & 54 & 0 \\
NT & 07 & 06 & 25 \\
TN & 21 & $60(48 \%)$ & $25(20 \%)$ \\
Total & $28(22 \%)$ & & \\
\hline
\end{tabular}

and $500 \mathrm{~ms}$ (40 units). Single neuron raster plots and population mean PSTHs of responses to oddball stimuli (Fig. $4 C)$ showed no responses to any of the sound tokens other than $S_{1}$ and $D$, again showing pure oddball detection like responses.

To consider the generality of the pure oddball detection with two narrowband sounds instead of one narrowband and one broadband, we also collected responses to oddball stimuli with two tones (TT; 102 units) but not necessarily in pairs as in the NT/TN cases. As with TN and NT cases, there was a lack of responses to the $S$ token beyond $S_{1}$ and a strong response to the $D$ tone (Fig. $4 D$ ). Comparing the mean DSI (Fig. 4E) among the three ways of computing selectivity index (considering $S_{X}$ in Eq. 3, to be the response to $S_{1}, S_{A L L}$, and $S_{P}$ ) showed no significant difference between $\mathrm{DSI}_{\mathrm{ALL}}$ and $\mathrm{DSI}_{\mathrm{P}}(0.49$ and 0.6 , NS, ANOVA). In a small subset of units $(n=25)$, for which paired data were available with the same stimulus parameters (repetition rate, position of D) as the TN and NT dataset, $\mathrm{CSI}_{\mathrm{ALL}}$ and $\mathrm{CSI}_{\mathrm{P}}$ were also not different $(0.23$ and 0.26 , NS, ANOVA). The comparatively lower CSI value could be because of lack of responses to some of the tones as $\mathrm{D}$ in this dataset or higher spontaneous rate, which also further shows strong adaptation to the $S$ tone. Thus, OFC neurons probed with oddball stimuli show that they inherently detect changes or violations to the regularity in the stimulus space and could be an important attribute required for flexible value updating in the OFC.

\section{Sparse responses in the OFC of awake, passively listening mice also show oddball detection}

To confirm that the observed deviance detection in the OFC is also present in neurons in awake condition, singleunit recordings in passively listening mice were performed with electrodes implanted in the left OFC. As in the anesthetized case, we found robust responses to auditory stimulation, with $73 \%$ (162/219) units showing excitatory responses to pure tones (Fig. $5 A, B$ ), which is a much higher proportion of neurons than in an earlier study (Winkowski et al., 2018).

Table 5: AC response types depending on the stimulus type and its location in the sequence

\begin{tabular}{lllll}
\hline & $\begin{array}{l}\mathrm{S}_{1}=1 \text { and } \\
\mathrm{D}=1 \\
\text { (units) }\end{array}$ & $\begin{array}{l}\mathrm{S}_{1}=1 \text { and } \\
\mathrm{D}=0 \\
\text { (units) }\end{array}$ & $\begin{array}{l}\mathrm{S}_{1}=0 \text { and } \\
\mathrm{D}=1 \\
\text { (units) }\end{array}$ & $\begin{array}{l}\mathrm{S}_{1}=0 \text { and } \\
\mathrm{D}=0 \\
\text { (units) }\end{array}$ \\
\hline $\begin{array}{c}\text { Tone as } \mathrm{S}_{1} \\
\text { or } \mathrm{D}\end{array}$ & 7 & 20 & 0 & 35 \\
$\begin{array}{c}\text { Noise as } \mathrm{S}_{1} \\
\text { or } \mathrm{D}\end{array}$ & 45 & 16 & 1 & 0 \\
\hline
\end{tabular}


A
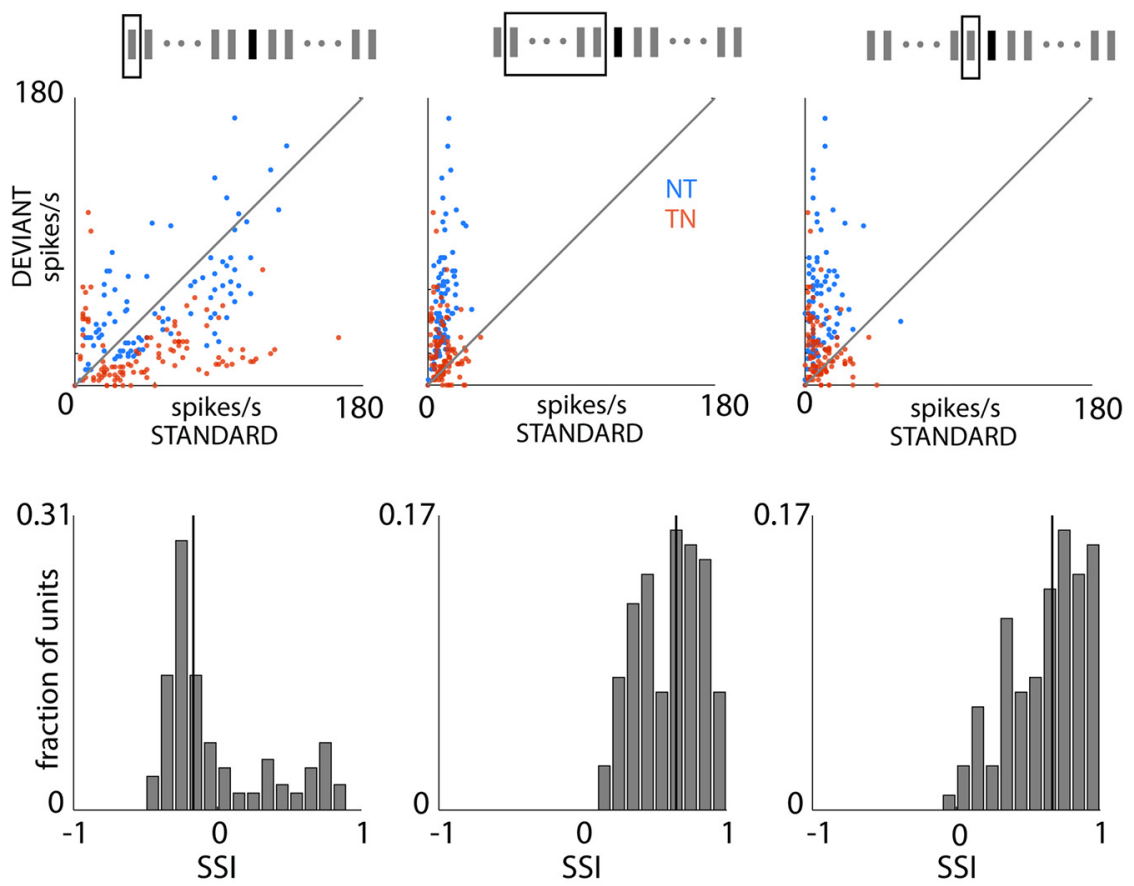

C
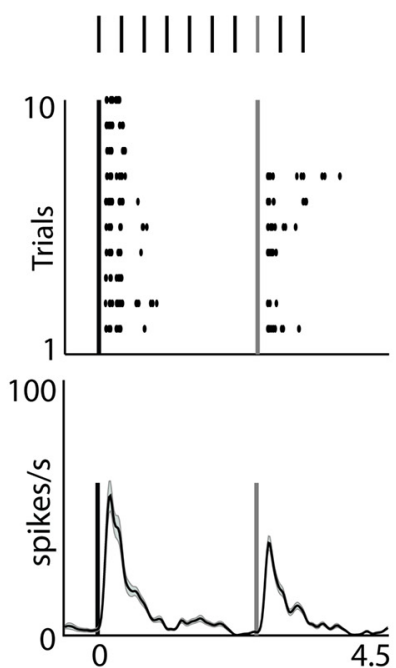
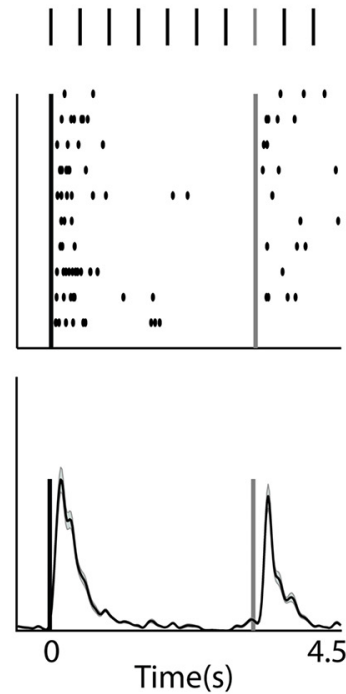
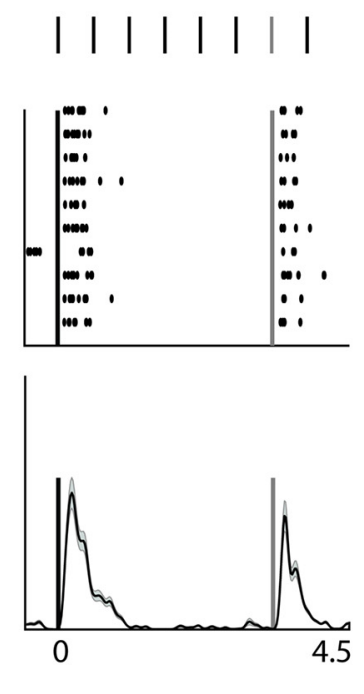

B

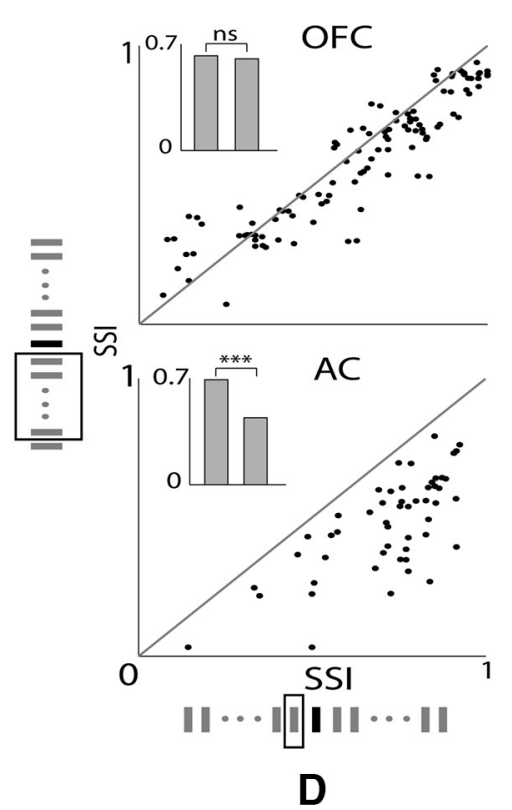

$25 \mid$ || || ||| ||||||||

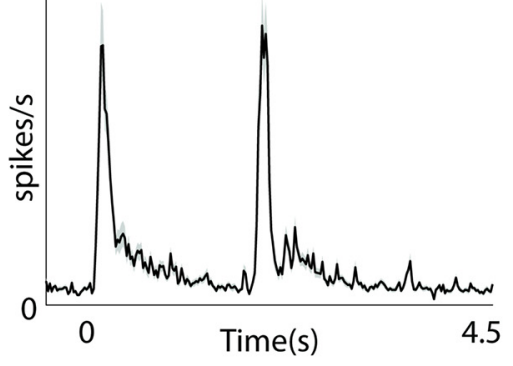

E

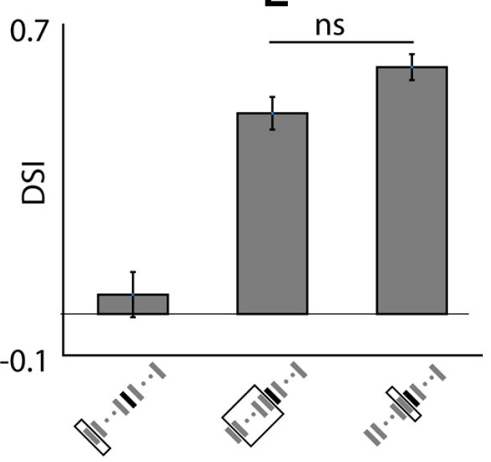

Figure 4. Pure oddball Detection in OFC unlike AC (ii). $\boldsymbol{A}$, top, Scatter plot of mean spike rate at standard and deviant in NT (blue) and TN (orange). The spike rates for standard were calculated either considering the $\mathrm{S}_{1}$ (left), or $\mathrm{S}_{\mathrm{ALL}}$ (middle), or $\mathrm{S}_{\mathrm{PT}}$ (right). The tokens considered in the two cases are enclosed in the gray rectangle. The histograms at the bottom show the CSls computed by using three different standards

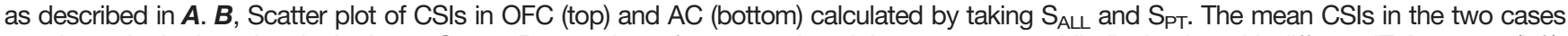
are shown in the bar plots in the inset. $\mathbf{C}$, top, Raster plots of an example unit in response to oddball stimulus with different ITol; $300 \mathrm{~ms}$ (left), $400 \mathrm{~ms}$ (middle), and $500 \mathrm{~ms}$ (right). Bottom, Mean population PSTH for these intervals. $\boldsymbol{D}$, Mean population PSTH to tone-tone oddball stimulus. $\boldsymbol{E}$, Mean DSI for tone-tone oddball stimulus with three different standards as described in $\boldsymbol{A}$. ns, not significant, ${ }^{\star \star \star} p<0.001$.

The neurons showed expectedly much shorter latency to peak (134.5 $\pm 2.83 \mathrm{~ms}$ SEM; Fig. 5 C) than in the anesthetized mice, while a small percentage (14\%) of units showed a very short latency $(\sim 14 \mathrm{~ms})$ and were not included in further analyses as they could be related to sound-evoked movement. Similar to anesthetized mice, a small fraction of neurons $(4 \%)$ got suppressed on presentation of tones in the awake state as well. 
A

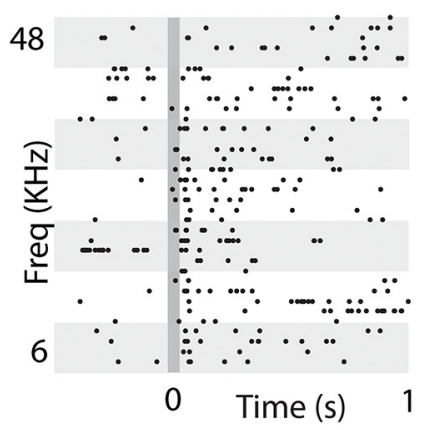

C

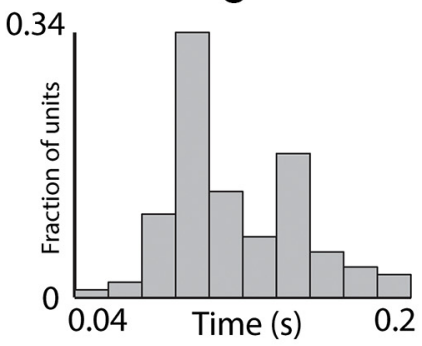

B

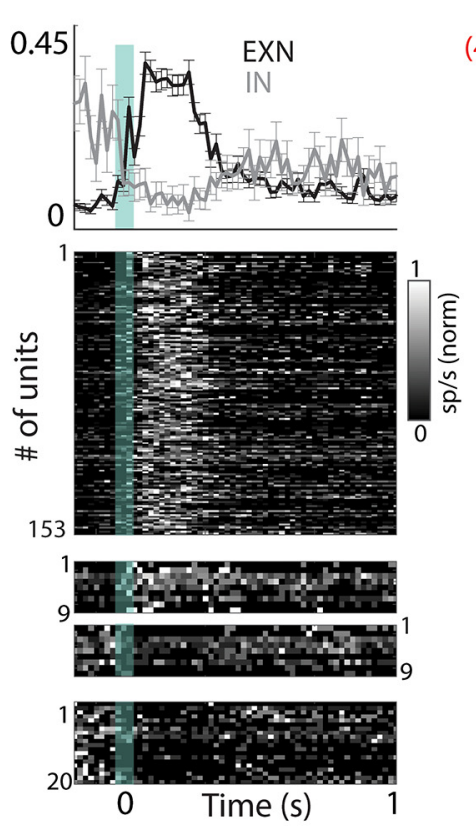

D
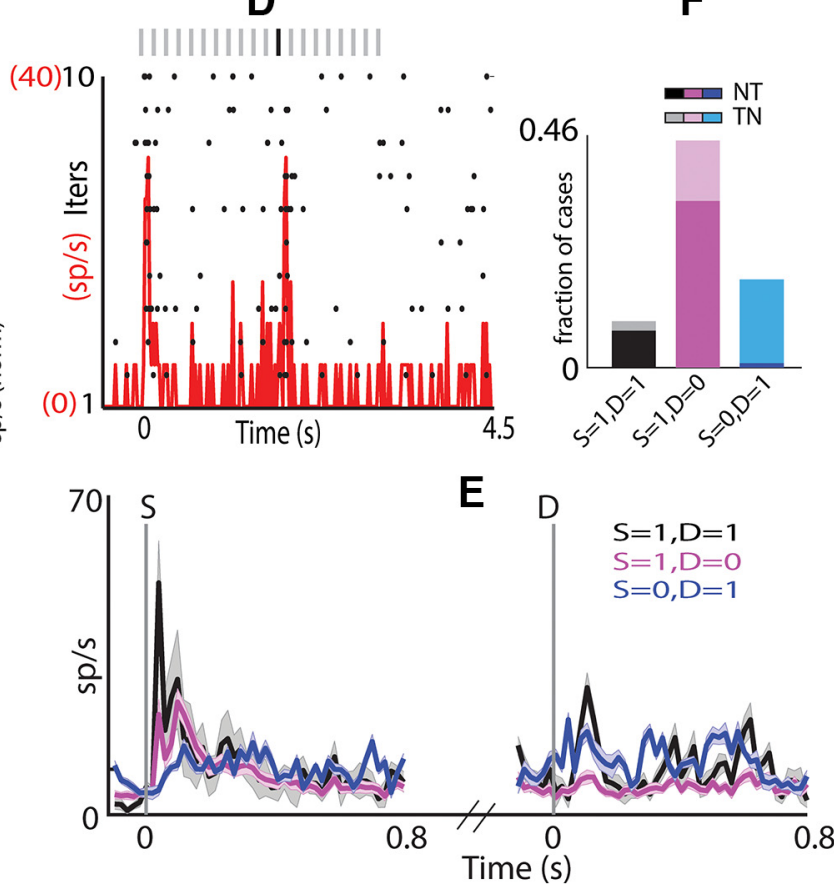

Figure 5. Awake mouse OFC also shows deviance detection. $\boldsymbol{A}$, Raster plot of an example unit in the OFC showing responses to tones of different frequencies in awake condition. $\boldsymbol{B}$, top, Mean normalized PSTH \pm SEM of all units showing excitation (black) and inhibition (gray) on auditory stimulation. Bottom, Individual unit's PSTH (upper panel: units showing excitation; middle two panels: units showing excitation to some frequency and inhibition to some other frequency; lower panel: units showing inhibition). $\boldsymbol{C}$, A distribution of peak response latency in the awake condition. $\boldsymbol{D}$, Raster plot (black) and PSTH (red) of an example unit showing responses to oddball stimulus. $\boldsymbol{E}$, Mean population PSTH \pm SEM of units responding to both standard onset and deviant $(S=1, D=1$; black), responding only to standard onset $(S=1, D=0$; magenta) and responding only to deviant $(S=0, D=1 ;$ blue). $\boldsymbol{F}$, Fraction of units belonging to different categories as described in $\boldsymbol{E}$. The darker shades show the fraction of units belonging to NT and lighter shades show fraction of units belonging to TN group.

In the awake head-fixed passively listening mice, responses to TN and NT pairs of oddball stimuli were collected from 54 units (108 cases; Fig. 5D,E) which responded to at least one of the four stimuli (two $S_{1}$ tokens and two D tokens), as earlier. The population PSTHs (Fig. 5E) shows that neurons responded only to the $S_{1}$ and then to the $\mathrm{D}$ token and not to the other tokens, as was observed in the anesthetized animals. Thus, the basic observation of immediate adaptation to a $S$ token and response only to the $D$ token, if at all, giving rise to deviant selectivity, is the same as in the anesthetized mice. However, the responses in the oddball case were far more selective, sparse and context dependent in the case of passively listening mice than in anesthetized mice. In 30/108 (28\%) cases, there were responses to neither $S_{1}$ nor $D$, which is significantly larger than that in the anesthetized OFC and AC (OFC: $34 / 218, p<0.01$ and AC: $12 / 124, p<0.001$, two proportion $z$ test). In 10/108 cases, there were responses to both $S_{1}$ and $D$ (eight NT and two TN), 49/108 cases to only $S_{1}$ (36 NT and $13 \mathrm{TN}$ ), and 19/108 cases to only the D token ( 1 NT and $18 \mathrm{TN}$; Fig. $5 F$ ). Thus, overall, in the awake condition, there were responses to the $D$ token in $27 \%(29 / 108)$ cases as compared with $53 \%$ cases in the anesthetized OFC (Fig. 3B), showing even higher selectivity of responses. When considering the same sound tokens as $\mathrm{S}$ and $\mathrm{D}$, unlike the anesthetized case, we found far stronger context dependence of responses, particularly to tones. In 1(14), 14(30), 8(6), and $31(4)$ out of 54 units, there were responses to tones (noise), both as $S_{1}$ and $D$, only as $S_{1}$ and not as $D$, only as $D$ and not as $S_{1}$, and to neither $S_{1}$ nor as $D$, respectively. Thus, the responses to tones were far sparser and selective in the oddball stimuli with only $43 \%(23 / 54)$ units showing responses to the tone (either as $S_{1}$ or as $\left.D\right)$, although for pure tones there were responses in $73 \%$ units $(p<0.001$, two proportion $z$ test).

\section{OFC receives the major projections from the dorsal AC but the major excitatory drive from the ventral AC}

To find the main source of auditory afferents in the mice OFC capable of driving auditory responses, we performed neuroanatomical experiments by injecting 200-nl green retrobeads (Lumafluor Inc) stereotactically in the OFC. We specifically targeted the location where we performed electrophysiological recordings ( $n=9$; Figs. $1 A, 6 A)$. The number of beads in the regions across medio-lateral (ML) extent encompassing the AuV, A1, and AuD were observed and quantified (Fig. 6B; Materials and Methods). In a subset of experiments, we injected anterograde $A A V$. CB7.Cl.mCherry in MGBv that allowed us to confirm the extent of $\mathrm{A} 1$ and $\mathrm{AuV}$ along the ML extent. Since both 
A

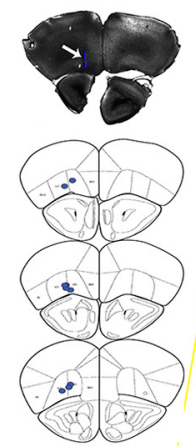

B
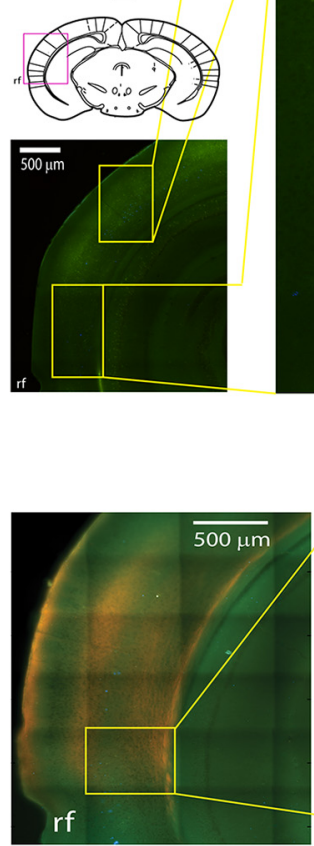

C

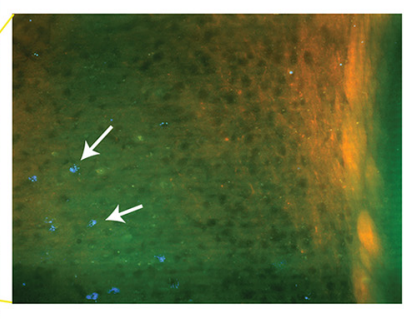

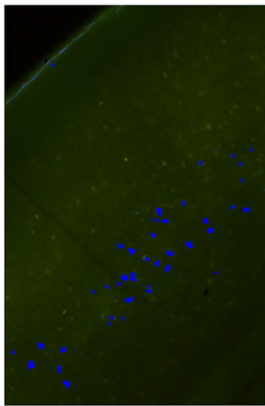
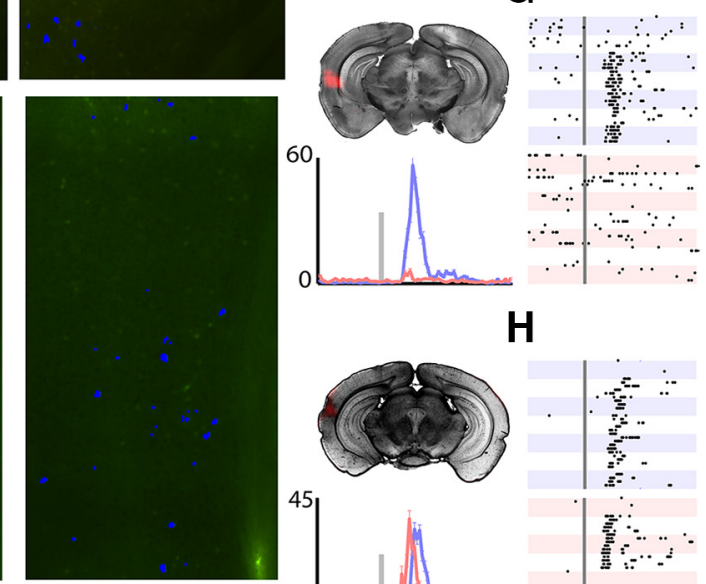
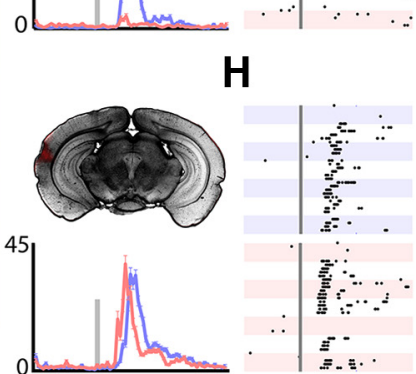

$\mathbf{F}$

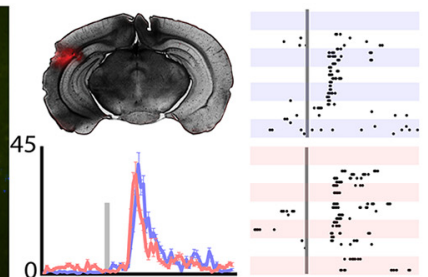

G

I
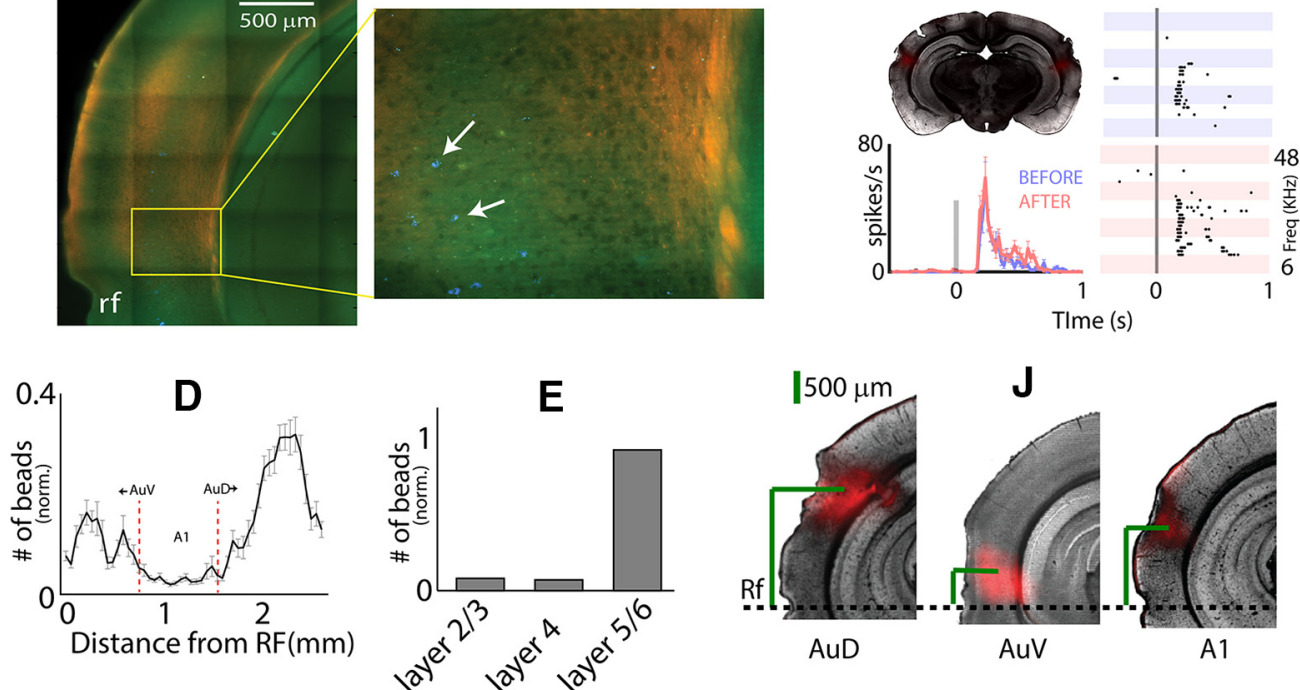

Figure 6. Distinct contributions of AC divisions in OFC auditory responses. $\boldsymbol{A}$, top, Coronal brain section showing retrobeads injection site (white arrow) in the OFC. Bottom: Injection sites in all nine animals. $\boldsymbol{B}$, top left, Coronal brain section from mouse atlas showing AC. Magenta box roughly marks the part of the brain region shown in bottom left image. Bottom left, Regions of AuD and AuV are marked in the yellow box. rf: rhinal fissure. Middle, AuD (top) and AuV (bottom) showing labeled cell bodies (white arrows) by the retrograde transported beads from OFC. Right, Same regions with enhanced intensities of the blue pixels for easy visualization of the beads. $\boldsymbol{C}$, left, Brain section showing layer 4 of the AC labeled with AAV-mcherry injected in the MGBv. Right, Zoomed-in image of the area inside the yellow box on the left showing beads. $\boldsymbol{D}$, Mean number of beads as a function of distance from the rf. Dashed red lines mark the extent of A1. $\boldsymbol{E}$, Laminar distribution of beads in the AC. $\boldsymbol{F}$, Brain section showing block site in the AuD; raster plots of an example unit before and after silencing the AuD; mean population PSTH \pm SEM before (blue) and after (red) silencing the AuD. Vertical gray line is the stimulus time. G, Brain section showing block site in the AuV; raster plots of an example unit before and after silencing the AuV; mean population PSTH \pm SEM before (blue) and after (red) silencing the AuV. $\boldsymbol{H}$, Brain section showing block site in the A1; raster plots of an example unit before and after silencing the A1; mean population PSTH \pm SEM before (blue) and after (red) silencing the A1. I, Brain section showing dual block sites in both A1; raster plots of an example unit before and after silencing both the A1s; mean population PSTH \pm SEM before (blue) and after (red) silencing both the A1s. J, Block sites in the AuD, AuV and A1 from the rf. 
AuV and A1 (Fig. $6 C$ ) receive MGBv projections (Ohga et al., 2018), the unlabeled region, dorsal to $A 1$, could be identified as AuD. The regions were demarcated in other mice (without MGBv-labeled projections) based on the mouse atlas (Paxinos and Franklin, 2013), which corresponded well with our observations in mice with MGBv injections. Additionally, labeled MGBv projections also allowed us to corroborate lamina specific distribution of cells projecting to the OFC. The average projection profile across the ML extent of AC showed that AuD had the highest density of cells projecting to the OFC, followed by AuV, with minimal projections from A1 (Fig. 6D). The lamina specific distributions (Fig. $6 E$ ) showed that most of the projections to OFC from the AC originated in the infragranular layers (Layer V/VI). Thus, with both A1/AuV and AuD projecting to the OFC, it is likely that both the lemniscal and non-lemniscal pathways are involved in shaping auditory responses in the OFC.

Next, we tested the functionality of projections from $A C$ to the OFC to determine the contribution of the different regions of $A C$ to the auditory responses in the OFC. In a series of experiments, single-unit recordings with responses to pure tones in the OFC were performed before and after pharmacological inactivation of AuD $(n=3)$, AuV $(n=5)$, and A1 $(n=7)$ with muscimol and baclofen (Materials and Methods). The site of inactivation was confirmed by tracking fluorescent SR101 (Fig. 6F-H, left top) mixed with the muscimol-baclofen solution.

Contrary to expectation, the inactivation of AuD (Fig. $6 F$ ) did not affect the single-unit OFC responses to auditory stimulation (Fig. $6 F$, right, example single-unit dot raster). The population mean PSTHs ( $n=45$ units) before and after AuD inactivation (Fig. 6F, bottom left), showed no difference (blue and red, mean spike rates $72.2 \pm 3.7$ and $65.7 \pm 4.8$, NS). Significant responses to all frequencies before inactivation were included in constructing the population PSTHs. While the AuV, with a lesser number of neurons than AuD projecting directly to the OFC $[0.11 \pm$ 0.02 and $0.2 \pm 0.03$ beads (normalized), $p<0.01 t$ test], was found to be the source of almost the entire auditorydriven excitatory activity in the OFC. Similar plots as before, of example dot raster and population mean PSTHs ( $n=43$ units) for before and after AuV inactivation (Fig. 6G) show that OFC auditory responses were almost completely abolished following AuV block $(98.7 \pm 3.3$ and $14.9 \pm 1.4, p<10^{-76}$ ). Of course, other than the direct inputs to OFC from AuV, other inputs to OFC providing such excitatory auditory inputs cannot be ruled out, but such indirect pathways also must originate from the AuV. Thus, the dorsal and ventral divisions of non-A1 are in stark contrast of each other in terms of their contribution to OFC auditory responses. However, their direct anatomic connections show characteristics opposite to their functional contributions. Similar to AuD block, the inactivation of $\mathrm{A} 1$ (Fig. $6 \mathrm{H}$ ) did not lead to any change in firing rates $(70.1 \pm 2.4$ and $72.9 \pm 3.4, \mathrm{NS})$, as observed in population mean PSTHs ( $n=62$ units) and dot raster plots (Fig. 6H). The possibility of a contralateral A1 contribution through indirect pathways to OFC was also considered.
Bilateral inactivation of A1 (Fig. 6/, top left) also did not change firing rates of OFC neurons as assessed through population mean PSTHs ( $n=30$ units) and mean firing rates of single units in response to tones before and after pharmacological inactivation of both A1s $(61.7 \pm 10.07$ and $79.5 \pm 11.3, \mathrm{NS})$.

\section{Auditory responses in the OFC originate from both, the lemniscal and non-lemniscal MGB}

Since $A 1$ and $A u D$ inactivation did not cause changes in response rates in the OFC and given that MGBv projects on AuV as well (Ohga et al., 2018), we hypothesized that OFC auditory responses originate in the lemniscal MGBv. To test the hypothesis, single-unit recordings in response to pure tones in the OFC were performed before and after MGBv inactivation (Fig. 7A). As with AuV inactivation, auditory responses in the OFC were completely abolished with MGBv block (23 units, $121.3 \pm 9.7$ and $18.5 \pm 1.5$, $p<10^{-16}$ ). Since MGBv efferents almost entirely project to A1/AAF and AuV, we conclude that the entire auditory driven excitatory input originates in the lemniscal auditory thalamus (MGBv). For our MGBv inactivation, we confirmed post hoc (Fig. $7 \mathrm{~A}$, top left, inset) the lack of SR101 in dorsal MGB (MGBd; Paxinos and Franklin, 2013) to rule out the inactivation of the neurons projecting to AuV from there. Physical damage to MGBd during GABA agonist injections to MGBv was also ruled out by repeating the experiments with saline injections to MGBv which did not alter tone response rates in the OFC ( $n=18$ units, $93 \pm 6$ and $75.6 \pm 6.6$, NS). Also, since AuD with major thalamic afferents from MGBd (Honma et al., 2013; Kok and Lomber, 2017) did not alter OFC responses, the involvement of the non-lemniscal MGBd in OFC auditory responses are at best minimal. The contribution of the MGBm to OFC auditory responses was also tested similarly (Fig. 7B). As opposed to abolition or no change in response rates observed in the other inactivation experiments, responses to pure tones altered dramatically in the OFC following inactivation of MGBm. Most units showed a behavior similar to the example single-unit activity before (blue) and after (red) MGBm block shown in Figure 7B, right. The population mean PSTHs (42 units) showed no change in peak response rates $(54.7 \pm 2.8$ and $52 \pm$ 2.8, NS, $t$ test) with MGBm inactivation, but the responses became persistent, with spiking continuing following the stimulus onset sometimes up to $1 \mathrm{~s}$ and mean response duration almost doubling $(177 \pm 6$ and $314 \pm 11 \mathrm{~ms}, p<0.001$; Fig. $7 C$ ). Thus, MGBm, which has multiple sources of auditory inputs as well as other sensory inputs, is a source of long-lasting auditory driven inhibition in the OFC affecting the temporal profile of auditory responses.

Given the strong connection from MGBm to LA (Woodson et al., 2000) and LA to BLA (Ledoux, 2000) and dense projections from BLA to OFC (Lichtenberg et al., 2017), we hypothesized that the major MGBm contribution to OFC auditory responses is through the BLA and the effects of BLA inactivation would produce an effect similar to that of MGBm silencing. As expected, on 
A

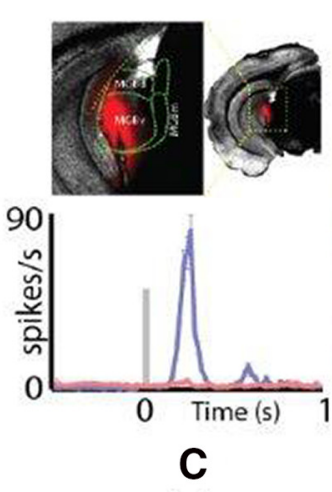

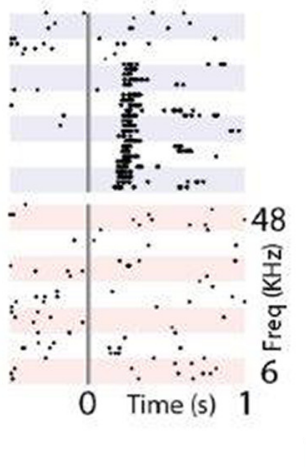

B

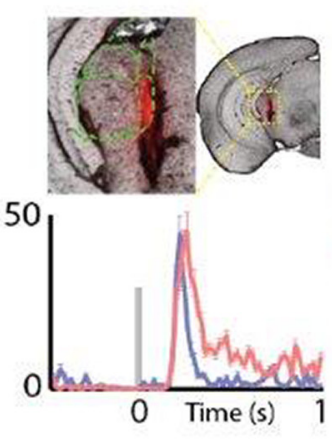

D

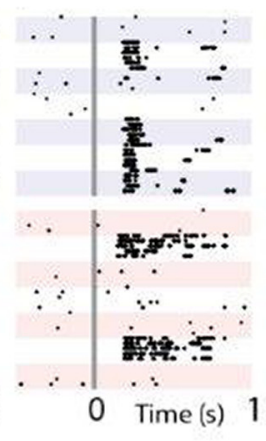

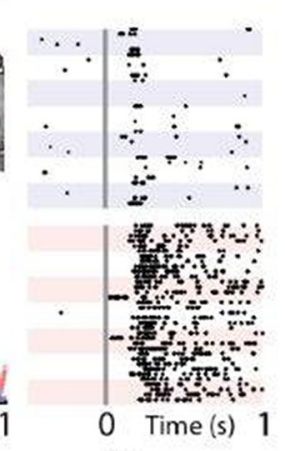

E
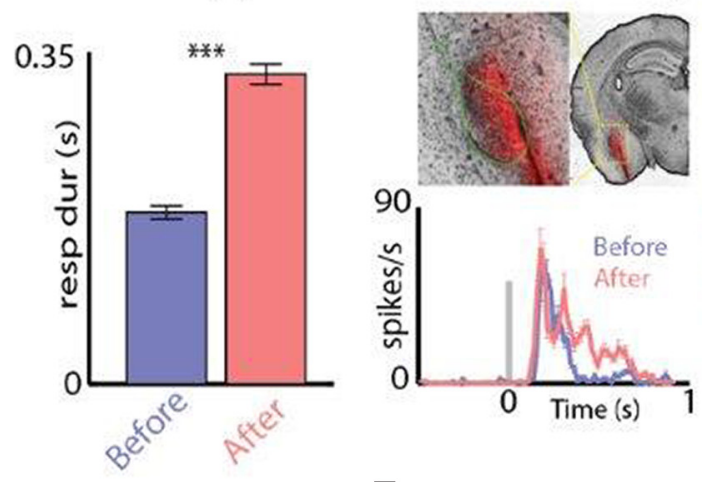

F
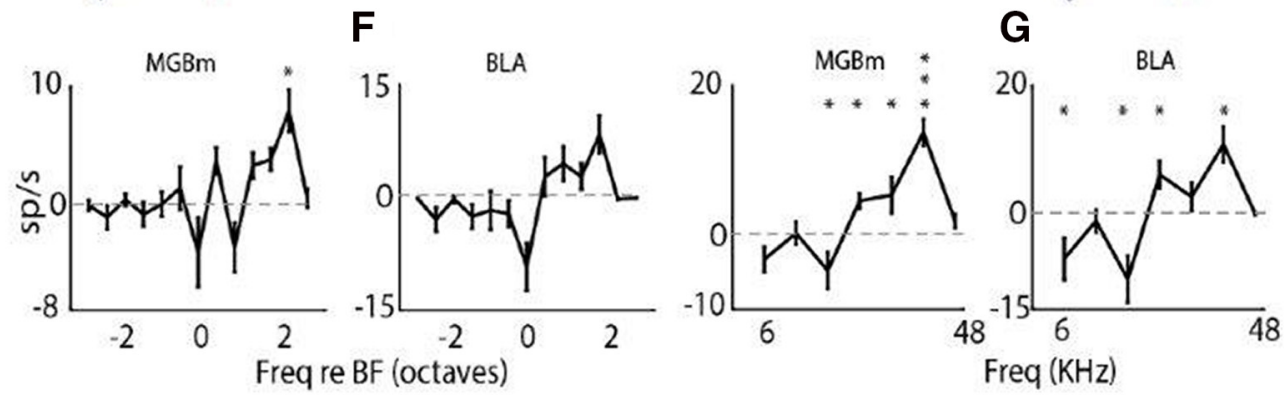

Figure 7. Parallel excitatory and inhibitory contributions to OFC auditory responses originate in the lemniscal and non-lemniscal auditory thalamic nuclei. $\boldsymbol{A}$, Brain section showing block site in the MGBv; raster plots of an example unit before and after silencing the MGBv; mean population PSTH \pm SEM before (blue) and after (red) silencing MGBv. Vertical gray line is the stimulus time. $\boldsymbol{B}$, Brain section showing block site in the MGBm; raster plots of an example unit before and after silencing the MGBm; mean population PSTH \pm SEM before (blue) and after (red) silencing the MGBm. $\boldsymbol{C}$, Mean response duration before and after silencing the MGBm. $\boldsymbol{D}$, Brain section showing block site in the BLA; raster plots of an example unit before and after silencing the BLA; mean population PSTH \pm SEM before (blue) and after (red) silencing the BLA. $E$, Mean response duration before and after silencing the BLA. Mean inhibitory inputs into OFC, from MGBm and BLA as a function of $(\boldsymbol{F})$ frequency with respect to BF and (G) absolute frequency. ${ }^{*} p<0.05,{ }^{\star \star *} p<0.001$.

inactivating the BLA (Fig. 7D), we found a similar response pattern with long-lasting persistent activity in the OFC (Fig. 7D, right, example dot raster), with no change in peak firing rates as assessed through the population mean PSTHs before and after BLA block (56 units, $68.7 \pm$ 4.6 and $78.9 \pm 5$, NS, $t$ test). As with MGBm block, response duration increased (Fig. 7E) by similar degrees with BLA inactivation (response duration: $156 \pm 11$ and $338 \pm 14, p<0.001, t$ test).

The change in response to tones after MGBm or BLA inactivation was quantified by considering the difference in mean rate responses (after-before) to the different frequencies (in the window of response duration after inactivation; Materials and Methods) relative to the BF of the units (Fig.
$7 F$, MGBm, block left, BLA block, right). In both cases, a similar pattern of rate difference was observed, with almost all frequency components relative to $\mathrm{BF}$, showing no significant difference except a peak at two octaves above BF and a negative peak at BF. Thus, the MGBm or BLA-based inhibition into the OFC is not organized in a BF-specific way. Rather when considering the changes in firing rate before and after MGBm or BLA inactivation (Fig. 7G, left and right) in absolute frequency, we found that significant inhibitory inputs are in the middle frequency region of mouse hearing $(17-34 \mathrm{kHz})$. Further, we observed that there is a trend of a significant net excitatory contribution mediated through both MGBm and BLA in the lower frequencies $(6-12 \mathrm{kHz})$. Since the pathway originating in the MGBm is associated 

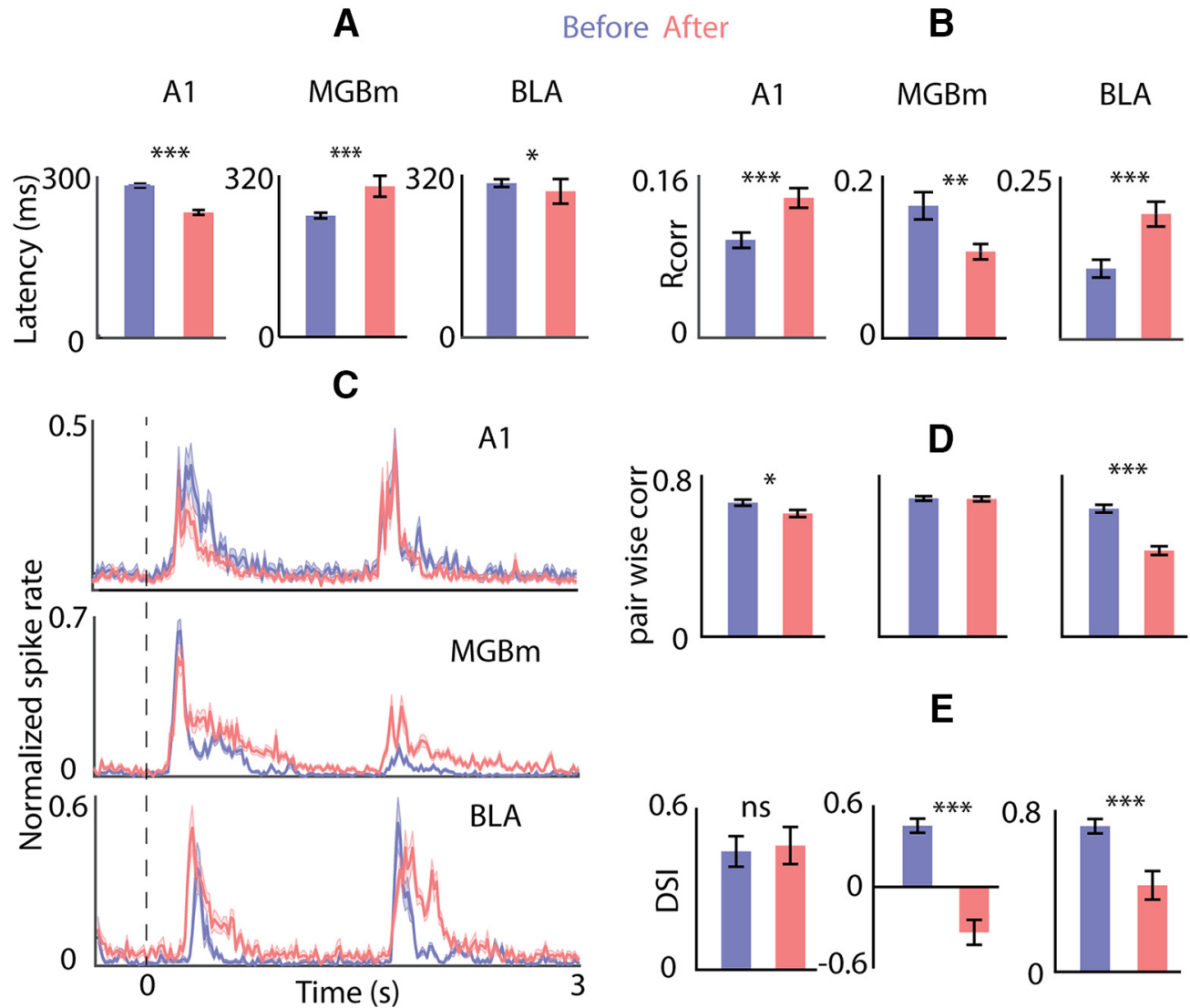

\section{E}

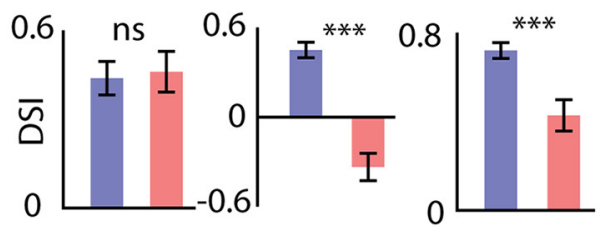

Figure 8. Both OFC deviant selectivity and spike time-based response properties are shaped by the non-lemniscal pathway unlike by A1. $\boldsymbol{A}$, Mean population latency \pm SEM to pure tones before (blue) and after (red) silencing A1 (left), MGBm (middle), and BLA (right). $\boldsymbol{B}$, Mean population reliability $\left(R_{\text {corr }}\right) \pm$ SEM before (blue) and after (red) silencing A1 (left), MGBm (middle), and BLA (right). $\boldsymbol{C}$, Mean population PSTH \pm SEM in response to oddball stimulus before (blue) and after (red) silencing A1 (top), MGBm (middle), and BLA (bottom). $\boldsymbol{D}$, Mean population pairwise correlations \pm SEM before (blue) and after (red) silencing A1 (left), MGBm (middle), and BLA (right). $\boldsymbol{E}$, Mean population DSI \pm SEM before (blue) and after (red) silencing A1 (left), MGBm (middle), and BLA (right). ${ }^{*} p<0.05,{ }^{* *} p<0.01,{ }^{* \star *} p<0.001$.

with fear conditioning, it is likely that this pathway is shaped based on the previous natural experience of fear associated stimuli and auditory events.

\section{Auditory response properties and deviant selectivity of OFC: contributions of auditory input sources}

With the inactivation of $\mathrm{A} 1$, although there are no changes in firing rate, there is a significant reduction in the latency of responses to tones in single units of the OFC $(306 \pm 3.4,258 \pm 4.33 \mathrm{~ms}, p<0.001$, paired $t$ test; Fig. $8 A$, left). Such latency reduction is usually associated with, a stimulus getting effectively stronger (for example, with an increasing sound level of noise; Fig. 1B), weaker long-term adaptation (Fig. 2E), or disinhibition. Similarly, on considering latencies before and after inactivation of MGBm and BLA, opposing effects of latency were found, although in both cases similar changes were observed in terms of response rates and response duration. MGBm inactivation led to a marked increase in latency to tones $(246.5 \pm 4.4,296.6 \pm 7.3 \mathrm{~ms}, p<0.001$, paired $t$ test; Fig. $8 A$, middle) while inactivation of BLA barely led to a reduction in latency $(315.4 \pm 7.4,297.4 \pm 8 \mathrm{~ms}$, NS $t$ test, removing the effect of outliers and comparing medians, $p<0.05$ rank-sum). Since the initial latency of response varied between the two populations of single units (before MGBm block and before BLA block), we also compared the fractional change in latency of single units on MGBm inactivation and BLA inactivation (mean $27 \%$ and $7 \%$ unpaired $t$ test $p<0.001$ ). Thus, the effective inhibition in the OFC, originating from MGBm is not simply relayed by the $B L A$. There are likely other auditory sources that also provide inhibition via BLA on to OFC. Such sources of inhibition on OFC through BLA, likely from AC (Bertero et al., 2019) remain intact on MGBm inactivation leading to longer latency, while with BLA inactivation their effect is removed to lead to the reduced latency.

Spike timing is crucial in generating synchronization in populations of neurons (Kreuz et al., 2007; Ermentrout et al., 2008), plasticity (Benedetti et al., 2009; Li et al., 2014), and forming associations (Atilgan et al., 2018). We considered spike timing reliability in the OFC single units before and after the inactivation of the different auditory sources. A1 inactivation remarkably increased spike timing reliability quantified through $R_{\text {corr }}$ (from $0.0953 \pm 0.01$ to $0.1385 \pm 0.01, p<0.001$, paired $t$ test; Fig. $8 B$, left) in response to tones. Although the absolute $\mathrm{R}_{\text {corr }}$ values were low, the increase on block was highly significant. Again, a 
differential effect was observed with BLA and MGBm inactivation. While MGBm inactivation decreased spike timing reliability (from $0.16 \pm 0.02$, to $0.11 \pm 0.01, p<0.01$, paired $t$ test; Fig. $8 B$, middle), BLA inactivation nearly doubled reliability of spike times (from $0.11 \pm 0.02$ to 0.19 $\pm 0.02, p<0.001$, paired $t$ test; Fig. $8 B$, right) in the repeated presentation of tones. Since inhibition is crucial in precise spike timing (Pouille and Scanziani, 2001; Wehr and Zador, 2003), and with MGBm having the highest reliability in spiking (Anderson and Linden, 2011) among the auditory thalamic nuclei, the loss of BLA mediated feedforward inhibition originating from MGBm, may lead to the decreased reliability is spiking in the OFC. However, since A1 acts as a source of spike timing jitter in the OFC (Fig. 8B, left), blocking of BLA also effectively removes the effect of A1 dominated spiking jitter introduced in the OFC through LA.

As our data suggest that context dependence and pure oddball detection are the hallmark of the auditory responses of the OFC, we used responses to oddball stimuli before and after inactivation to further understand the contribution of the different auditory sources to response properties of the OFC (Fig. $8 \mathrm{C}$ ). Pairwise correlations can strengthen coding efficiency (Hung et al., 2015) and enhance plasticity (Feldman, 2009). We compared synchronization of responses in pairs of simultaneously recorded OFC single units before and after inactivation of each of the auditory sources. Inactivation of A1 produced a small decrease in synchronization with oddball stimuli (from $0.67 \pm 0.01$ to $0.61 \pm 0.02, p<0.05, t$ test; Fig. $8 D$, left). However, MGBm inactivation caused no change in pairwise correlations $(0.69 \pm 0.01$ and $0.69 \pm 0.01$, NS; Fig. $8 D$, middle), while BLA inactivation reduced pairwise correlations (from $0.64 \pm 0.02$ to $0.43 \pm 0.02, p<0.001 t$ test; Fig. $8 D$, right). Thus, the effective inhibitory input to the OFC from the BLA synchronizes activity across populations of single units, independent of the MGBm input to the BLA. As BLA inactivation increases spike timing reliability of single neurons, it suggests that auditory driven indirect AC input to the OFC via BLA causes synchronization across pairs of neurons. The above is further corroborated by the fact that $A 1$ inactivation reduced pairwise correlations in the OFC, although to a lesser degree.

Finally, we consider the contribution of different auditory sources on the oddball detection property of OFC single units. Since we did not have sufficient data on pairs of NT and TN both before and after inactivation of different structures, we considered DSI instead of CSI to quantify oddball selectivity. Population mean PSTHs of OFC single units in response to oddball stimuli before and after inactivation of $A 1, M G B m$, and BLA (Fig. 8C, each row) show the emergence of persistent activity at both the onset and the deviant except in the case of $A 1$ block. Quantification of the selectivity (DSI) before and after block shows that $A 1$ does not contribute to DSI in the OFC, while inactivation of both the MGBm and the BLA produced a drastic reduction in DSI (from $0.44 \pm 0.06$ to $0.46 \pm 0.07, p=0.57,0.46 \pm 0.05$ to $-0.34 \pm 0.09$, $p<0.001$, and $0.72 \pm 0.04$ to $0.43 \pm 0.07, p<0.001$, respectively, $t$ test; Fig. $8 E$ ). The difference in the reduction of DSI in case of MGBm and BLA inactivation suggests that the primary source of the selectivity to oddball stimuli in the OFC is the MGBm, while it is further strengthened by auditory inputs from the BLA from sources other than MGBm (like AC) to the OFC. However, it should be noted that with neither MGBm nor BLA inactivation, responses to subsequent tokens after the onset token appeared. Thus, although values of DSI reduce on average to produce lower oddball selectivity in both cases (MGBm and BLA inactivation), the OFC's intrinsic selectivity to the deviant and fast adaptation to subsequent stimuli over a long timescale is unchanged. AuV, the primary excitatory input to the OFC, itself does not show similar deviant selectivity and adaptation (Fig. 3D).

\section{Discussion}

We find the presence of robust auditory responses in single units of the mouse OFC during passive listening both in awake as well as in the anesthetized state. Auditory responses in the OFC as in AC showed robust responses to tones and noise, the usual stimuli used for characterization. Other studies probing the OFC with different kinds of auditory stimuli in mice (Winkowski et al., 2018) and primates (Rolls et al., 2006) have also found robust responses. However, in our study, we show that the OFC auditory responses were drastically different from $A C$ in several major ways which have not been studied in the OFC previously like (1) absence of topographical organization; (2) longer history dependence and adaptation; and (3) presence of pure oddball detection. Although AC auditory responses are also affected by multiple timescales of adaptation (Ulanovsky et al., 2004), OFC neurons showed extremely long history dependence and adaptation lasting above $10 \mathrm{~s}$ as observed with comparisons of OFC and A1/AuV responses with a variety of ITIs (Fig. 2). Since the outcome of a stimulus is almost always temporally offset by large durations (Pavlov, 1927; Fuster and Alexander, 1971), this long history dependence in the OFC may potentially encode the sensory aspect of the auditory memory over long timescales and may play a key role in creating various stimulus-outcome associations (Delamater, 2007; Rudebeck et al., 2008; Sadacca et al., 2018) and their revaluation. The OFC neurons show pure oddball detection during oddball stimulus streams, ceasing to respond from the first instant of repetition of the standard stimulus. This degree of faster and stronger adaptation to repetition is not seen in AC and appears to emerge in the OFC possibly achieved by local circuits within OFC through recurrence (Yarden and Nelken, 2017).

In our pharmacological block experiments, we found that $\mathrm{A} 1$ does not contribute to OFC response strength but only induces more jitter in spike timing and longer response latency. This effect could be via an early inhibition through a weak input along the A1/AuV-LA-BLA (Romanski et al., 1993; Ledoux, 2000; Tsukano et al., 2019) or directly from $A 1$ to OFC pathways (Fig. $6 D, H$ ). Through anatomic tracing, we found that secondary areas of the $A C$ send most of the projections to OFC within $A C$ with AuD showing the strongest labeling. Despite the 
strongest labeling, the OFC responses were not affected on inactivating AuD. The mouse AuD is more involved in representing the perceptual meaning of primarily temporally structured sounds while AuV is thought to represent value in terms of novelty (Weible et al., 2014; Geissler et al., 2016). The OFC is involved in stimulus outcome value computation, which is consistent with the result that AuV drives excitatory auditory responses in the OFC. AuD, on the other hand, is likely recruited to provide inputs to the OFC in a more behavioral context-specific manner with ultrasonic complex vocalizations (Tsukano et al., 2015), multimodal stimuli (Morrill and Hasenstaub, 2018), or multimodal spatial tuning (Bizley and King, 2009). The function and necessity of the AuD projections on to the OFC require further investigation. Since OFC responses were abolished on silencing MGBv as with inactivation of AuV which receives direct inputs from the MGBv (Fig. 7A; Ohga et al., 2018), we hypothesize that the OFC auditory responses are driven by MGBv via AuV.

We also show that auditory inputs to the OFC originate in at least two parallel regions in the MGB, the ventral and medial divisions. These two streams converge in at least two locations, the amygdala, and the OFC. The inputs from AuV carry in sensory information with context dependence which is sharpened through the long-lasting deviant selective inhibitory drive originating from the MGBm and modified in the BLA and also through local recurrent connections in the OFC. Both MGBm and BLA could provide a saliency filtering of the sensory inputs to the OFC and suppress responses in OFC units following deviant/salient auditory events. The MGBm and BLA also provide a means of controlling the sensory driven activity by causing persistent activity in the OFC which may allow the sensory stimulus to be associated with other outcome related delayed signals related to reward (Thorpe et al., 1983; Hikosaka and Watanabe, 2000; Stalnaker et al., 2014), prediction error (O'Neill and Schultz, 2013), or punishment (O'Doherty et al., 2001; Windmann et al., 2006) required for reinforcement in acquisition as well as in reversal learning. Such longer persistent activity is seen in the PFC during working memory related behavior (Fuster and Alexander, 1971; Funahashi et al., 1989; Curtis and D'Esposito, 2003) and also in the OFC conveying incentive value of cues (Gallagher et al., 1999; Tremblay and Schultz, 1999). A study showed the presence of a group of neurons in the MGBm that silences itself during conditioned stimulus presentation (O'Connor et al., 1997). $M G B m$ is the first station in the auditory pathway that shows changes in the firing rate because of task-related stimulus associations (Birt et al., 1979) and forms part of the thalamo-amygdaloid component in the auditory pathway (Ledoux et al., 1986) crucial in fear learning. We also show that frequency profile of the inhibitory inputs from the MGBm via the LA-BLA-OFC pathway is innately tuned to the $30-\mathrm{kHz}$ region (Fig. $7 \mathrm{G}$ ), which is also the frequency range of fear-induced vocalizations. Thus, the MGBm driven control of OFC activity is likely to do with associations of fear eliciting stimuli (Weinberger et al., 1995; Weinberger, 2011).

Silencing BLA causes decorrelation of response in pairs of OFC units and increase in spike timing precision across trials, other than causing persistence and lowering of deviant detection. Precisely timed spiking in the persistent OFC activity may aid in plasticity (Markram et al., 1997; Bi and Poo, 1998; Dan and Poo, 2004; Feldman, 2009) required for associating the auditory stimulus with delayed outcome related signals. Similarly, decorrelation of activity, removing redundancies in the population, allows more possibilities of creating associations with the OFC stimulus driven activity and outcome signals. BLA is known to encode valence (Paton et al., 2006; Janak and Tye, 2015; Zhang and $\mathrm{Li}, 2018$ ) and damage to BLA-OFC connections may lead to disruption of decision-making and reversal learning like behaviors (Zeeb and Winstanley, 2013; Orsini et al., 2015; Lichtenberg et al., 2017; Groman et al., 2019). We hypothesize that these disruptions may arise because of lack of deviant selectivity and precise inhibitory control imposed by the BLA affecting the required persistent activity. Thus, we propose that the MGBm via LA-BLA and BLA itself act as controllers of persistent activity required for stimulus-outcome associations during behaviors like decision-making and reversal learning.

A limitation of our study is that the experiments revealing the contributions of different auditory areas to the OFC responses were conducted in the anesthetized animals. Hence the behavioral significance of these findings could not be established. Besides, the effects of indirect pathways to the OFC that may get affected on silencing an area cannot be ruled out with the current experiments.

\section{References}

Anderson LA, Linden JF (2011) Physiological differences between histologically defined subdivisions in the mouse auditory thalamus. Hear Res 274:48-60.

Atilgan H, Town SM, Wood KC, Jones GP, Maddox RK, Lee AKC, Bizley JK (2018) Integration of visual information in auditory cortex promotes auditory scene analysis through multisensory binding. Neuron 97:640-655.e4.

Benedetti BL, Glazewski S, Barth AL (2009) Reliable and precise neuronal firing during sensory plasticity in superficial layers of primary somatosensory cortex. J Neurosci 29:11817-11827.

Bertero A, Feyen PLC, Zurita H, Apicella AJ (2019) A non-canonical cortico-amygdala inhibitory loop. J Neurosci 39:8424-8438.

Bi GQ, Poo MM (1998) Synaptic modifications in cultured hippocampal neurons: dependence on spike timing, synaptic strength, and postsynaptic cell type. J Neurosci 18:10464-10472.

Birt D, Nienhuis R, Olds M (1979) Separation of associative from non-associative short latency changes in medial geniculate and inferior colliculus during differential conditioning and reversal in rats. Brain Res 167:129-138.

Bizley JK, King AJ (2009) Visual influences on ferret auditory cortex. Hear Res 258:55-63.

Chen IW, Helmchen F, Lütcke H (2015) Specific early and late oddball-evoked responses in excitatory and inhibitory neurons of mouse auditory cortex. J Neurosci 35:12560-12573.

Curtis CE, D'Esposito M (2003) Persistent activity in the prefrontal cortex during working memory. Trends Cogn Sci 7:415-423.

Dan Y, Poo MM (2004) Spike timing-dependent plasticity of neural circuits. Neuron 44:23-30.

Delamater AR (2007) The role of the orbitofrontal cortex in sensoryspecific encoding of associations in Pavlovian and instrumental conditioning. Ann NY Acad Sci 1121:152-173.

Dilgen J, Tejeda HA, O'Donnell P (2013) Amygdala inputs drive feedforward inhibition in the medial prefrontal cortex. J Neurophysiol 110:221-229. 
Ermentrout GB, Galán RF, Urban NN (2008) Reliability, synchrony and noise. Trends Neurosci 31:428-434.

Feldman DE (2009) Synaptic mechanisms for plasticity in neocortex. Annu Rev Neurosci 32:33-55.

Fresno V, Parkes SL, Faugère AL, Coutureau E, Wolff M (2019) A thalamocortical circuit for updating action-outcome associations. Elife 8:e46187.

Fritz JB, David SV, Radtke-Schuller S, Yin P, Shamma SA (2010) Adaptive, behaviorally gated, persistent encoding of task-relevant auditory information in ferret frontal cortex. Nat Neurosci 13:10111019.

Funahashi S, Bruce CJ, Goldman-Rakic PS (1989) Mnemonic coding of visual space in the monkey's dorsolateral prefrontal cortex. J Neurophysiol 61:331-349.

Fuster JM, Alexander GE (1971) Neuron activity related to short-term memory. Science 173:652-654.

Gallagher M, McMahan RW, Schoenbaum G (1999) Orbitofrontal cortex and representation of incentive value in associative learning. J Neurosci 19:6610-6614.

Geissler DB, Schmidt HS, Ehret G (2016) Knowledge about soundscontext-specific meaning differently activates cortical hemispheres, auditory cortical fields, and layers in house mice. Front Neurosci 10:98.

Groman SM, Keistler C, Keip AJ, Hammarlund E, DiLeone RJ, Pittenger C, Lee D, Taylor JR (2019) Orbitofrontal circuits control multiple reinforcement-learning processes. Neuron 103:734-746. e3.

Hikosaka K, Watanabe M (2000) Delay activity of orbital and lateral prefrontal neurons of the monkey varying with different rewards. Cereb Cortex 10:263-271.

Honma Y, Tsukano H, Horie M, Ohshima S, Tohmi M, Kubota Y, Takahashi K, Hishida R, Takahashi S, Shibuki K (2013) Auditory cortical areas activated by slow frequency-modulated sounds in mice. PLoS One 8:e68113.

Hung CP, Cui D, Chen YP, Lin CP, Levine MR (2015) Correlated activity supports efficient cortical processing. Front Comput Neurosci 8:171.

Janak PH, Tye KM (2015) From circuits to behaviour in the amygdala. Nature 517:284-292.

Kok MA, Lomber SG (2017) Origin of the thalamic projection to dorsal auditory cortex in hearing and deafness. Hear Res 343:108117.

Kreuz T, Haas JS, Morelli A, Abarbanel HDI, Politi A (2007) Measuring spike train synchrony. J Neurosci Methods 165:151161.

Ledoux JE (2000) Emotion circuits in the brain. Annu Rev Neurosci 23:155-184.

Ledoux JE, Sakaguchi A, Iwata J, Reis DJ (1986) Interruption of projections from the medial geniculate body to an archi-neostriatal field disrupts the classical conditioning of emotional responses to acoustic stimuli. Neuroscience 17:615-627.

Lee CC (2015) Exploring functions for the non-lemniscal auditory thalamus. Front Neural Circuits 9:69.

Lee D, Seo H, Jung MW (2012) Neural basis of reinforcement learning and decision making. Annu Rev Neurosci 35:287-308.

Li XY, Wang N, Wang YJ, Zuo ZX, Koga K, Luo F, Zhuo M (2014) Long-term temporal imprecision of information coding in the anterior cingulate cortex of mice with peripheral inflammation or nerve injury. J Neurosci 34:10675-10687.

Lichtenberg NT, Pennington ZT, Holley SM, Greenfield VY, Cepeda X, Levine MS, Wassum KM (2017) Basolateral amygdala to orbitofrontal cortex projections enable cue-triggered reward expectations. J Neurosci 37:8374-8384.

Luna VM, Morozov A (2012) Input-specific excitation of olfactory cortex microcircuits. Front Neural Circuits 6:1-7.

Markram H, Lübke J, Frotscher M, Sakmann B (1997) Regulation of synaptic efficacy by coincidence of postsynaptic APs and EPSPs. Science 275:213-215.

McGarry LM, Carter AG (2016) Inhibitory gating of basolateral amygdala inputs to the prefrontal cortex. J Neurosci 36:9391-9406.
Miller EK, Cohen JD (2001) An integrative theory of prefrontal cortex function. Annu Rev Neurosci 24:167-202.

Morrill RJ, Hasenstaub AR (2018) Visual information present in infragranular layers of mouse auditory cortex. J Neurosci 38:28542862.

Morrison SE, Salzman CD (2011) Representations of appetitive and aversive information in the primate orbitofrontal cortex. Ann NY Acad Sci 1239:59-70.

Nieto-Diego J, Malmierca MS (2016) Topographic distribution of stimulus-specific adaptation across auditory cortical fields in the anesthetized rat. PLoS Biol 14:e1002397.

O'Connor KN, Allison TL, Rosenfield ME, Moore JW (1997) Neural activity in the medial geniculate nucleus during auditory trace conditioning. Exp Brain Res 113:534-556.

O’Doherty J, Kringelbach ML, Rolls ET, Hornak J, Andrews C (2001) Abstract reward and punishment representations in the human orbitofrontal cortex. Nat Neurosci 4:95-102.

O'Neill M, Schultz W (2013) Risk prediction error coding in orbitofrontal neurons. J Neurosci 33:15810-15814.

Ohga S, Tsukano H, Horie M, Terashima H, Nishio N, Kubota Y, Takahashi K, Hishida R, Takebayashi H, Shibuki K (2018) Direct relay pathways from lemniscal auditory thalamus to secondary auditory field in mice. Cereb Cortex 28:4424-4439.

Orsini CA, Trotta RT, Bizon JL, Setlow B (2015) Dissociable roles for the basolateral amygdala and orbitofrontal cortex in decision-making under risk of punishment. J Neurosci 35:1368-1379.

Ostlund SB, Balleine BW (2007) Orbitofrontal cortex mediates outcome encoding in pavlovian but not instrumental conditioning. $J$ Neurosci 27:4819-4825.

Paton JJ, Belova MA, Morrison SE, Salzman CD (2006) The primate amygdala represents the positive and negative value of visual stimuli during learning. Nature 439:865-870.

Pavlov IP (1927) Conditioned reflexes: an investigation of the physiological activity of the cerebral cortex. Oxford: Oxford University Press.

Paxinos G, Franklin KBJ (2013) Paxinos and Franklin's the mouse brain in stereotaxic coordinates, Ed 4, San Diego: Academic Press.

Pouille F, Scanziani M (2001) Enforcement of temporal fidelity in pyramidal cells by somatic feed-forward inhibition. Science 293:1159-1163.

Rolls ET, Critchley HD, Browning AS, Inoue K (2006) Face-selective and auditory neurons in the primate orbitofrontal cortex. Exp Brain Res 170:74-87.

Romanski LM, Clugnet MC, Bordi F, LeDoux JE (1993) Somatosensory and auditory convergence in the lateral nucleus of the amygdala. Behav Neurosci 107:444-450.

Rudebeck PH, Behrens TE, Kennerley SW, Baxter MG, Buckley MJ, Walton ME, Rushworth MFS (2008) Frontal cortex subregions play distinct roles in choices between actions and stimuli. J Neurosci 28:13775-13785.

Sacco T, Sacchetti B (2010) Role of secondary sensory cortices in emotional memory storage and retrieval in rats. Science 329:649656.

Sadacca BF, Wied HM, Lopatina N, Saini GK, Nemirovsky D, Schoenbaum G (2018) Orbitofrontal neurons signal sensory associations underlying model-based inference in a sensory preconditioning task. Elife 7:e30373.

Sawatari H, Tanaka Y, Takemoto M, Nishimura M, Hasegawa K, Saitoh K, Song W-J (2011) Identification and characterization of an insular auditory field in mice. Eur J Neurosci 34:1944-1952.

Schoenbaum G, Setlow B (2001) Integrating orbitofrontal cortex into prefrontal theory: common processing themes across species and subdivisions. Learn Mem 8:134-147.

Schoenbaum G, Roesch M (2005) Orbitofrontal cortex, associative learning, and expectancies. Neuron 47:633-636.

Schreiber S, Fellous JM, Whitmer D, Tiesinga P, Sejnowski TJ (2003) A new correlation-based measure of spike timing reliability. Neurocomputing 52-54:925-931. 
Slater BJ, Sons SK, Yudintsev G, Lee CM, Llano DA (2019) Thalamocortical and intracortical inputs differentiate layer-specific mouse auditory corticocollicular neurons. J Neurosci 39:256-270.

Stalnaker TA, Cooch NK, McDannald MA, Liu TL, Wied H, Schoenbaum G (2014) Orbitofrontal neurons infer the value and identity of predicted outcomes. Nat Commun 5:3926.

Taaseh N, Yaron A, Nelken I (2011) Stimulus-specific adaptation and deviance detection in the rat auditory cortex. PLoS One 6:e23369.

Thorpe SJ, Rolls ET, Maddison S (1983) The orbitofrontal cortex: neuronal activity in the behaving monkey. Exp Brain Res 49:93115.

Tremblay L, Schultz W (1999) Relative reward preference in primate orbitofrontal cortex. Nature 398:704-708.

Tsukano $\mathrm{H}$, Horie $\mathrm{M}$, Bo $\mathrm{T}$, Uchimura $\mathrm{A}$, Hishida R, Kudoh $\mathrm{M}$, Takahashi K, Takebayashi H, Shibuki K (2015) Delineation of a frequency-organized region isolated from the mouse primary auditory cortex. J Neurophysiol 113:2900-2920.

Tsukano H, Hou X, Horie M, Kitaura H, Nishio N, Hishida R, Takahashi K, Kakita A, Takebayashi H, Sugiyama S, Shibuki K (2019) Reciprocal connectivity between secondary auditory cortical field and amygdala in mice. Sci Rep 9:19610.

Ulanovsky N, Las L, Nelken I (2003) Processing of low-probability sounds by cortical neurons. Nat Neurosci 6:391-398.

Ulanovsky N, Las L, Farkas D, Nelken I (2004) Multiple time scales of adaptation in auditory cortex neurons. J Neurosci 24:1044010453.

Wallis JD (2007) Orbitofrontal cortex and its contribution to decisionmaking. Annu Rev Neurosci 30:31-56.

Wehr M, Zador AM (2003) Balanced inhibition underlies tuning and sharpens spike timing in auditory cortex. Nature 426:442-446.

Weible AP, Liu C, Niell CM, Wehr M (2014) Auditory cortex is required for fear potentiation of gap detection. J Neurosci 34:15437-15445.
Weinberger NM (2011) The medial geniculate, not the amygdala, as the root of auditory fear conditioning. Hear Res 274:61-74.

Weinberger NM, Javid R, Lepan B (1995) Heterosynaptic long-term facilitation of sensory-evoked responses in the auditory cortex by stimulation of the magnocellular medial geniculate body in guinea pigs. Behav Neurosci 109:10-17.

Wilson RC, Takahashi YK, Schoenbaum G, Niv Y (2014) Orbitofrontal cortex as a cognitive map of task space. Neuron 81:267-279.

Windmann S, Kirsch P, Mier D, Stark R, Walter B, Güntürkün O, Vaitl $D(2006)$ On framing effects in decision making: linking lateral versus medial orbitofrontal cortex activation to choice outcome processing. J Cogn Neurosci 18:1198-1211.

Winkowski DE, Bandyopadhyay S, Shamma SA, Kanold PO (2013) Frontal cortex activation causes rapid plasticity of auditory cortical processing. J Neurosci 33:18134-18148.

Winkowski DE, Nagode DA, Donaldson KJ, Yin P, Shamma SA, Fritz JB, Kanold PO (2018) Orbitofrontal cortex neurons respond to sound and activate primary auditory cortex neurons. Cereb Cortex 28:868-879.

Woodson W, Farb CR, Ledoux JE (2000) Afferents from the auditory thalamus synapse on inhibitory interneurons in the lateral nucleus of the amygdala. Synapse 38:124-137 .

Yarden TS, Nelken I (2017) Stimulus-specific adaptation in a recurrent network model of primary auditory cortex. PLoS Comput Biol 13:e1005437.

Zeeb FD, Winstanley CA (2013) Functional disconnection of the orbitofrontal cortex and basolateral amygdala impairs acquisition of a rat gambling task and disrupts animals' ability to alter decisionmaking behavior after reinforcer devaluation. J Neurosci 33:64346443.

Zhang X, Li B (2018) Population coding of valence in the basolateral amygdala. Nat Commun 9:5195. 